\title{
Langmuir Turbulence in Swell
}

\author{
JAMES C. MCWilliams, EdWARd HuCKLE, AND JunHONG LiAng \\ Department of Atmospheric and Oceanic Sciences, University of California, Los Angeles, Los Angeles, California \\ PETER P. SUlLivan \\ National Center for Atmospheric Research, Boulder, Colorado
}

(Manuscript received 29 May 2013, in final form 14 November 2013)

\begin{abstract}
The problem is posed and solved for the oceanic surface boundary layer in the presence of wind stress, stable density stratification, equilibrium wind-waves, and remotely generated swell-waves. The addition of swell causes an amplification of the Lagrangian-mean current and rotation toward the swell-wave direction, a fattening of the Ekman velocity spiral and associated vertical Reynolds stress profile, an amplification of the inertial current response, an enhancement of turbulent variance and buoyancy entrainment rate from the pycnocline, and - for very large swell—an upscaling of the coherent Langmuir circulation patterns. Implications are discussed for the parameterization of Langmuir turbulence influences on the mean current profile and the material entrainment rate in oceanic circulation models. In particular, even though the turbulent kinetic energy monotonically increases with wave amplitude inversely expressed by the turbulent Langmuir number La, the Lagrangian shear eddy viscosity profile $\kappa^{L}(z)$ is a nonmonotonic function of La, first increasing with increasing wave amplitude up to approximately the wind-wave equilibrium level, then decreasing with additional swell-wave amplitude. In contrast, the pycnocline entrainment rate is a monotonic function $\sim \mathrm{La}^{-2}$.
\end{abstract}

\section{Introduction}

The wind blows and the waves rise and roll on. This is the regime of Langmuir turbulence in the oceanic surface boundary layer (BL), so-called because Langmuir circulations (often recognized by the windrows in the surfactants they cause) are the primary turbulent eddies whose vertical momentum and buoyancy fluxes maintain the mean ageostrophic current and density stratification. Langmuir circulations arise from the instability of wind-driven boundary layer shear in the presence of Stokes drift (Craik and Leibovich 1976; McWilliams et al. 1997). Alternatively expressed, this regime is a wind-driven Ekman layer with stable interior density stratification and surface gravity waves that induce wave-averaged vortex and Coriolis forces and buoyancy advection due to the Lagrangian-mean Stokes drift velocity. Langmuir turbulence is common in nature (Belcher 2012).

Corresponding author address: James C. McWilliams, Dept. of Atmospheric and Oceanic Sciences, UCLA, 405 Hilgard Ave., Los Angeles, CA 90095-1565.

E-mail: jcm@atmos.ucla.edu
The physics of this regime is now well understood, primarily on the basis of many large eddy simulation (LES) studies of the wind-wave equilibrium state [e.g., the review in Sullivan and McWilliams (2010)]. However, in nature the wind is rarely in equilibrium with the waves (Young 1999; Sullivan et al. 2008; Hanley et al. 2010). Often this is due to transient wind changes or limited fetch, when the waves have not yet evolved into a fully developed equilibrium but are headed there over an interval of hours or over an offshore distance of tens of kilometers. Disequilibrium also occurs when swell-waves with a large amplitude and long wavelength propagate thousands of kilometers away from their generation in an earlier strong storm to a place with weaker local wind whose wind-waves are weaker and shorter. During their propagation, swell waves exhibit slow decay and slow evolution through dispersion and four-wave resonant interaction, and they have weak nonlinear interactions with the wind-waves encountered en route as long as there is significant separation in wavelength and/or direction (Masson 1993; Zakharov 2005; Depley et al. 2010). Under these conditions, a local quasi-equilibrium state of linearly superimposed swelland wind-waves can persist for a day or more if the wind 
is steady. These circumstances are especially common in the tropics and subtropics in both hemispheres, with swell-waves coming from higher-latitude winter storms (Alves 2006; Hanley et al. 2010). Also, they can occur anywhere in the lull after a storm.

Several idealizations of persistent disequilibrium have been examined with LES. Commonly, the wave spectrum is simplified to a monochromatic wave at the spectrum peak (McWilliams et al. 1997), the wind-wave Stokes drift is multiplied by a factor (usually less than one, implying weaker waves with a turbulent Langmuir number $\mathrm{La} \geq$ 0.3 , its equilibrium value; see section 2) (Harcourt and D'Asaro 2008; Grant and Belcher 2009), or the windwave Stokes drift direction is rotated relative to wind alignment (Van Roekel et al. 2012). All of these formulations are ad hoc compared to realistic persistent wind regimes where the wind-sea evolves toward equilibrium. On the other hand, the general formulation of wind-wave disequilibrium is daunting in the complexity of possible transient histories. In contrast, the case of equilibrium wind-waves with added steady swell-waves with wellseparated spectrum peak wavenumbers (hence $\mathrm{La}<0.3$ ) is an apt idealization of realistic persistent situations. Because the remote swell-generating storm and the local wind are independent, the added swell component can have an arbitrary orientation and amplitude relative to the local wind-sea. This is the problem addressed here.

\section{Formulation}

The LES code solves the wave-averaged dynamical equations in Sullivan and McWilliams (2010). These are incompressible, rotating Boussinesq fluid dynamics with a prognostic turbulent kinetic energy equation for subgrid-scale fluxes. Both model components have surface wave effects that include Stokes drift Coriolis and vortex forces and scalar advection, augmented pressure head, and subgrid Stokes drift energy production and advection. The "wind" forcing is by parameterized breakers that inject a body force $A$ and subgrid work $W$; for simplicity in the present paper, ensemble-mean breaker forcing profiles $\langle A\rangle(z)$ and $\langle W\rangle(z)$ are used, rather than stochastic individual impulses. (Angle brackets indicate a horizontal and temporal average, and $z$ is the vertical coordinate.) The same dynamical formulation is used in McWilliams et al. (2012) for the wavy Ekman layer with uniform density and equilibrium wind-waves. In particular, in that paper the differences between ensemble and stochastic breaker forcing are analyzed, as well as their differences compared with a conventional surface stress forcing with an equivalent integral effect (i.e., $\int\langle A\rangle d z=\tau / \rho_{o}=u_{*}^{2}$ in wind-wave equilibrium, where $\tau$ is stress, $\rho_{o}$ is the mean density, and $u *$ is the oceanic friction velocity). Apart from the level of turbulent energy and differences in the profiles of mean current and Reynolds stress very near the surface, this distinction in the representation of the breakers is not important for the topics of this paper. In this paper, $\tau$ and its associated $\langle A\rangle(z)$ are calculated from the near-surface wind speed $U_{a}$ with a bulk drag law; ${ }^{1}$ that is, $\tau=$ $\rho_{a} C_{D} U_{a}^{2}$, with $\rho_{a}=1 \mathrm{~kg} \mathrm{~m}^{-3}$ and $C_{D}=1.3$ for the cases here with $U_{a} \leq 10 \mathrm{~m} \mathrm{~s}^{-1}$.

The LES model is spun up from rest and approximately equilibrates over a period of a day or so, while retaining inertial oscillations that slowly decay over many days. In the present problem, an initial mean temperature stratification $T(z)$ is included. It initially has a well-mixed layer in the top $33 \mathrm{~m}$ and an interior gradient of $0.1^{\circ} \mathrm{Cm}^{-1}$ (implying a buoyancy frequency of $N=0.014 \mathrm{~s}^{-1}$ ). Turbulent entrainment develops, and the boundary layer deepens. Because this $N$ value is rather large, the boundary layer depth $h(t)$ only slowly deepens while the average mixed layer temperature $\bar{T}(t)$ cools in the absence of a surface heat flux. The Coriolis frequency $f$ is $10^{-4} \mathrm{~s}^{-1}$ (corresponding to a latitude of $45^{\circ} \mathrm{N}$ ) and is assumed to be spatially uniform over the small spatial scale of the domain. With the traditional approximation that neglects the horizontal projection of Earth's rotation vector, the solutions are symmetric with respect to wind direction; hence, without loss of generality, the wind direction is chosen as $\hat{\mathbf{x}}$ [east (E)] and held steady. The wind-sea spectrum is in equilibrium with the wind according to the prescription of Alves et al. (2003). The associated profiles for the Stokes drift velocity $\mathbf{u}_{\mathrm{st}}^{w}(z)$ and breaker forcings are calculated as in Sullivan et al. (2007). The $\mathbf{u}_{\mathrm{st}}^{w}$ is aligned with the wind direction.

The preceding formulation yields familiar solution behaviors that are described in several papers cited above. The novel aspect here is an added swell-wave component to the Stokes drift, which is modeled as

$$
\mathbf{u}_{\mathrm{st}}^{s}=U^{s} \exp \left[\frac{z}{D^{s}}\right] \hat{\boldsymbol{\theta}}^{s}
$$

for $z \leq 0$ where mean sea level is $z=0$. Because swellwave spectra are narrow, the swell is represented as a linear, monochromatic surface gravity wave with period $P$, wavelength $\lambda$, and amplitude $a$. The dispersion relation in deep water is $P=\sqrt{2 \pi \lambda / g}$ ( $g$ is gravitational acceleration). The associated Stokes drift has a depth scale $D^{s}=g P^{2} / 8 \pi^{2}$ and a surface velocity $U^{s}=8 \pi^{3} a^{2} / g P^{3}$.

\footnotetext{
${ }^{1}$ A more accurate drag law should include the influence of swell waves on $C_{D}$ for low wind speed (Sullivan et al. 2008).
} 

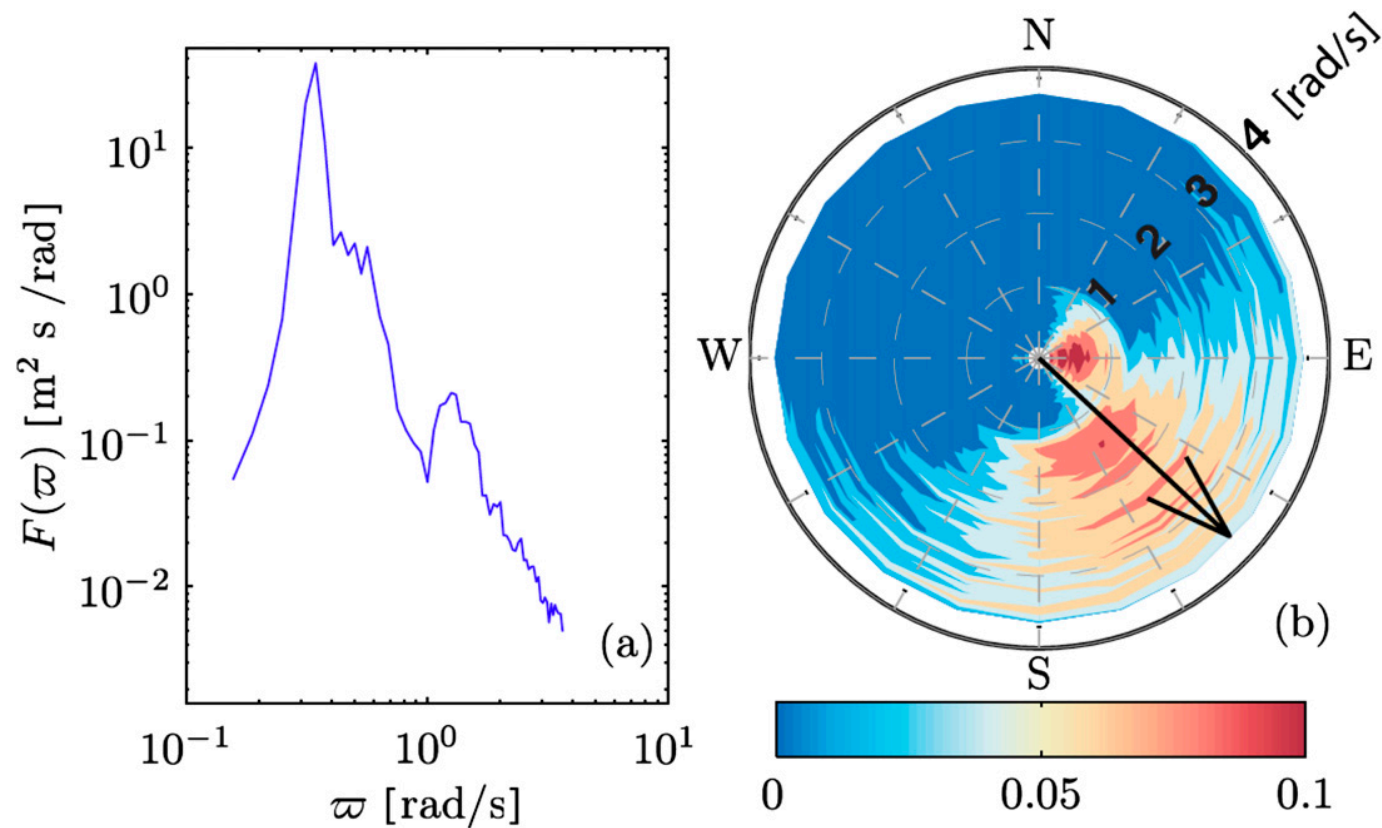

FIG. 1. Wave spectra during mixed wind and swell-wave conditions at the Harvest buoy (near Point Conception, California) from CDIP at 0030 UTC 5 Dec 2007. (left) Azimuthally integrated frequency spectrum for sea surface elevation $F(\bar{\omega})$, and (right) normalized directional spectrum $D(\bar{\omega}, \phi)=G(\bar{\omega}, \phi) / F(\bar{\omega})$, where $G$ is the directional spectrum (note that its $\phi$ integral is unity for each $\bar{\omega})$. The $\bar{\omega}\left(\mathrm{rad} \mathrm{s}^{-1}\right)$ is the wave frequency, and $\phi$ is the wave propagation direction. The simultaneous $U_{a}$ is $12 \mathrm{~m} \mathrm{~s}^{-1}$ in the direction of the black arrow, measured at the closest National Data Buoy Center station 46054. The wind-sea has a peak $P$ of about $4.5 \mathrm{~s}$ to the SE, and the swell wave has a $P$ of $19 \mathrm{~s}$ to the E. The $H_{s}$ is $6.9 \mathrm{~m}$.

The term $\hat{\boldsymbol{\theta}}^{s}$ is the unit direction vector for swell-wave propagation. The total Stokes drift is the sum of the windsea and swell contributions assuming no nonlinear interaction between their spectral components; that is,

$$
\mathbf{u}_{\mathrm{st}}(z)=\mathbf{u}_{\mathrm{st}}^{w}(z)+\mathbf{u}_{\mathrm{st}}^{s}(z) \text {. }
$$

Wave measurements are routinely made from oceanic buoys, and an extensive compendium is available from the Coastal Data Information Program (CDIP; www. cdip.ucsd.edu). Data and plots are presented in terms of $P($ or $\bar{f}=1 / P=\bar{\omega} / 2 \pi)$ and significant wave height $H_{s}$ $(=2 \sqrt{2} a$ for a monochromatic wave with sea level amplitude $a$ ). In some analyses waves with a spectrum peak $P$ longer than $10 \mathrm{~s}$ are categorized as swell-waves, although they are wind-waves in the midst of high winds; this ambiguity is rarer at subtropical and tropical sites with fewer big storms. Climatological histograms for individual sites are presented for the joint distribution of $P$ and $H_{s}$. Events with $H_{s}>10 \mathrm{~m}$ or $P>20 \mathrm{~s}$ are found, although the bulk of the swell-wave events are not so extreme. These imply possible ranges of $U^{s}$ up to $0.4 \mathrm{~m} \mathrm{~s}^{-1}$ and $D^{s}$ up to $50 \mathrm{~m}$, both of which are much larger than the amplitude and depth scale for Stokes drift in a moderate wind-sea. No restriction is placed on the swell-wave direction. Figure 1 shows a particular example of mixed wind- and swell-waves with large $H_{s}$.

LES solutions are calculated in a vertical domain $H=$ $100 \mathrm{~m}$ thick and a horizontally periodic domain (of width $L=300 \mathrm{~m}$, except where otherwise stated). The vertical grid is stretched with a smallest spacing of $0.3 \mathrm{~m}$ near the surface. The horizontal grid is uniform with a spacing of $2 \mathrm{~m}$. These grids encompass the boundary layer turbulence down to the resolution limit where the subgrid parameterization regularizes the flow.

The influence of swell-waves is explored through its parameters $U^{s}, D^{s}$, and $\hat{\theta}^{s}$ (Table 1 ). In the parameter survey case sets, these are varied independently; however, in nature $U^{s}$ and $D^{s}$ tend to be anticorrelated because of their inverse dependencies on powers of the period $P$ [see just below (1)]. The LES results are nondimensionalized by $u_{*}$ and by $h$ diagnosed from the solution averaged over the statistical analysis period $t=$ $0.55-1.18 \times 10^{5}$ s (i.e., one inertial period, $2 \pi / f$, with a Coriolis frequency $\left.f=10^{-4} \mathrm{~s}^{-1}\right)$. The term $h(t)$ is defined as the depth at which the mean thermal gradient $\partial_{z}\langle T\rangle(z)$ becomes as large as $0.05^{\circ} \mathrm{Cm}^{-1}$ just below the well-mixed layer. Initially, $h(0)=33 \mathrm{~m}$ in all cases.

An important parameter for Langmuir turbulence is the so-called Langmuir number: 
TABLE 1. Case sets analyzed in this paper. In all cases $\mathbf{u}_{\mathrm{st}}^{w}$ and $(A, W)$ are in equilibrium with the eastward wind. For these case sets, the La values are $0.14-0.24$ (Us1), 0.13-0.29 (Us2), 0.16 (Ds), 0.16-0.49 ( $\Theta \mathrm{s}), 0.11-0.16(\mathrm{Ua})$, and $0.11(\mathrm{Lh})$. In addition, there is a comparison case NB (i.e., No Stokes drift velocity and ensemblemean Breaker forcing) with $U_{a}=10 \mathrm{~m} \mathrm{~s}^{-1}$ and $\mathbf{u}_{\mathrm{st}}=0(\mathrm{La}=\infty)$.

\begin{tabular}{cc}
\hline \hline Case set & Wind and swell-wave parameters \\
\hline Us1 & $U_{a}=10 \mathrm{~m} \mathrm{~s}^{-1} ; D^{s}=16 \mathrm{~m} ; \theta^{s}=0 ; U^{s}=0-0.4 \mathrm{~m} \mathrm{~s}^{-1}$ \\
$\mathrm{Us} 2$ & $U_{a}=5 \mathrm{~m} \mathrm{~s}^{-1} ; D^{s}=16 \mathrm{~m} ; \theta^{s}=0 ; U^{s}=0-0.25 \mathrm{~m} \mathrm{~s}^{-1}$ \\
Ds & $U_{a}=10 \mathrm{~m} \mathrm{~s}^{-1} ; D^{s}=4-24 \mathrm{~m}(P=6-14 \mathrm{~s}) ; \theta^{s}=0 ;$ \\
& $U^{s}=0.25 \mathrm{~m} \mathrm{~s}^{-1}$ \\
$\Theta \mathrm{s}$ & $U_{a}=10 \mathrm{~m} \mathrm{~s}^{-1} ; D^{s}=16 \mathrm{~m} ; \theta^{s}=-\pi-+\pi ;$ \\
& $U^{s}=0.25 \mathrm{~m} \mathrm{~s}^{-1}$ \\
$\mathrm{Ua}$ & $U_{a}=2.5-10 \mathrm{~m} \mathrm{~s}^{-1} ; D^{s}=16 \mathrm{~m} ; \theta^{s}=0 ;$ \\
& $U^{s}=0.25 \mathrm{~m} \mathrm{~s}^{-1}$ \\
$\mathrm{Lh}$ & $U_{a}=2.5 \mathrm{~m} \mathrm{~s}^{-1} ; D^{s}=16 \mathrm{~m} ; \theta^{s}=0 ; U^{s}=0.25 \mathrm{~m} \mathrm{~s}^{-1} ;$ \\
& $L=300-1200 \mathrm{~m}$
\end{tabular}

$$
\mathrm{La}=\left[\frac{u_{*}}{\left|\mathbf{u}_{\mathrm{st}}(0)\right|}\right]^{1 / 2}
$$

[sometimes called the turbulent La to distinguish it from the laminar La defined for viscously controlled flows; Leibovich (1983)]. Small La is associated with large waves, especially large swell waves. Here, the surface value of $\mathbf{u}_{\mathrm{st}}$ is evaluated at the first grid level of $z=$ $-0.15 \mathrm{~m}$. The value of La is weakly grid dependent because there is a logarithmic singularity in $\mathbf{u}_{\mathrm{st}}^{w}$, as $z \rightarrow 0$ arising from the high-frequency tail in the wind-sea spectrum of Alves et al. (2003) with $F(\bar{f}) \propto \bar{f}^{-4}$. However, Romero and Melville (2010) show that the tail steepens to $F \propto \bar{f}^{-5}$ at sufficiently large $\bar{f}$ because of saturation, thus regularizing $\mathbf{u}_{\mathrm{st}}^{w}(0)$ on a vertical scale finer than resolved here. The swell-wave $\mathbf{u}_{\mathrm{st}}^{s}$ in (1) is smooth as $z \rightarrow 0$. Notice that a vector misalignment between swell- and wind-waves causes a reduction in $\left|\mathbf{u}_{\mathrm{st}}(0)\right|$, hence an increase in La, compared to alignment. Depending on the swell amplitude and orientation, La may or may not be increased compared to the case of only wind-waves.

\section{Analytic Ekman-Stokes model}

A partial interpretation of the LES results in sections 4 and 5 comes from an analytic solution of a wave-modified extension of the classic Ekman layer model, which is now called the Ekman-Stokes model. It represents a horizontal and time average of the horizontal momentum balance (14) with three simplifying assumptions: constant density $\rho_{o}$, constant eddy viscosity $\kappa_{o}$, and surface wind stress $\tau$ instead of breaker impulse $\mathbf{A}$ :

$$
f \hat{\mathbf{z}} \times\left(\langle\mathbf{u}\rangle+\mathbf{u}_{\mathrm{st}}\right)=\kappa_{o} \partial_{z}^{2}\langle\mathbf{u}\rangle,
$$

with a surface boundary condition of $\kappa_{o} \partial_{z}\langle\mathbf{u}\rangle(0)=\tau / \rho_{o}=$ $u_{*}^{2}$ and a lower boundary condition of $\mathbf{u} \rightarrow 0$ as $z \rightarrow-\infty$.
As is commonly done for analytic convenience, this is rewritten as a scalar equation for the complex velocity $\mathcal{U}=\langle u\rangle+i\langle v\rangle$ :

$$
\text { if }\left(\mathcal{U}+\mathcal{U}_{\mathrm{st}}\right)=\kappa_{o} \partial_{z}^{2} \mathcal{U}
$$

with boundary conditions of $\kappa_{o} \partial z \mathcal{U}(0)=\mathcal{T} \equiv\left(\tau^{x}+i \tau^{y}\right) /$ $\rho_{o}$ and $\mathcal{U}(-\infty)=0$. The problem is further simplified by assuming that the Stokes drift (2) has a monochromatic wind-wave component (identified with the wind-wave spectrum peak), structurally analogous to the swellwave component (1), so that

$\mathcal{U}_{\mathrm{st}} \equiv u_{\mathrm{st}}+i v_{\mathrm{st}}=U^{w} \exp \left[\frac{z}{D^{w}}\right]+U^{s} \exp \left[i \theta^{s}\right] \exp \left[\frac{z}{D^{s}}\right]$.

To match the problem posed in section 2, $T, U^{w}$, and $U^{s}$ are taken to be real and positive. Also, assume that $f>0$ (Northern Hemisphere).

Using simple analytic techniques, the Ekman-Stokes solution is derived:

$$
\begin{aligned}
\mathcal{U}= & \mathcal{U}_{e} \exp \left[\frac{(1+i) z}{h_{e}}\right]+\frac{i f U^{w}}{\kappa_{o} / D^{w 2}-i f} \exp \left[\frac{z}{D^{w}}\right] \\
& +\frac{i f U^{s} \exp \left[i \theta^{s}\right]}{\kappa_{o} / D^{s 2}-i f} \exp \left[\frac{z}{D^{s}}\right],
\end{aligned}
$$

with Ekman depth $h_{e}=\sqrt{2 \kappa_{o} / f}$ and amplitude coefficient

$\mathcal{U}_{e}=\frac{1-i}{\sqrt{2 f \kappa_{o}}}\left\{\mathcal{T}-\frac{i \kappa_{o} f U^{w}}{D^{w}\left(\kappa_{o} / D^{w 2}-i f\right)}-\frac{i \kappa_{o} f U^{s} \exp \left[i \theta^{s}\right]}{D^{s}\left(\kappa_{o} / D^{s 2}-i f\right)}\right\}$.

The transport integrals are

$$
\begin{aligned}
& \int_{-\infty}^{0} \mathcal{U} d z=-\frac{i \mathcal{T}}{f}-D^{w} U^{w}-D^{s} U^{s} \exp \left[i \theta^{s}\right], \\
& \int_{-\infty}^{0} \mathcal{U}_{\mathrm{st}} d z=D^{w} U^{w}+D^{s} U^{s} \exp \left[i \theta^{s}\right], \quad \text { and } \\
& \int_{-\infty}^{0} \mathcal{U}^{L} d z=-\frac{i \mathcal{T}}{f},
\end{aligned}
$$

where the complex Lagrangian velocity is $\mathcal{U}^{L}=\mathcal{U}+\mathcal{U}_{\text {st }}$ (i. e. , $\mathbf{u}^{L}=\langle\mathbf{u}\rangle+\mathbf{u}_{\text {st }}$ in vector notation). The Lagrangian transport is rotated $90^{\circ}$ to the right of the wind stress, while the Eulerian transport also has anti-Stokes components.

For the usual large swell situation of $U^{s}>U^{w}$ and $D^{s}>D^{w}$, these solutions indicate that a sufficient condition for the local wind-wave influence to be relatively unimportant in the boundary layer flow profile is for $U^{s} D^{s} \gg U^{w} D^{w}$. All of the cases in Table 1 with $U^{s} \neq 0$ are in this regime. 

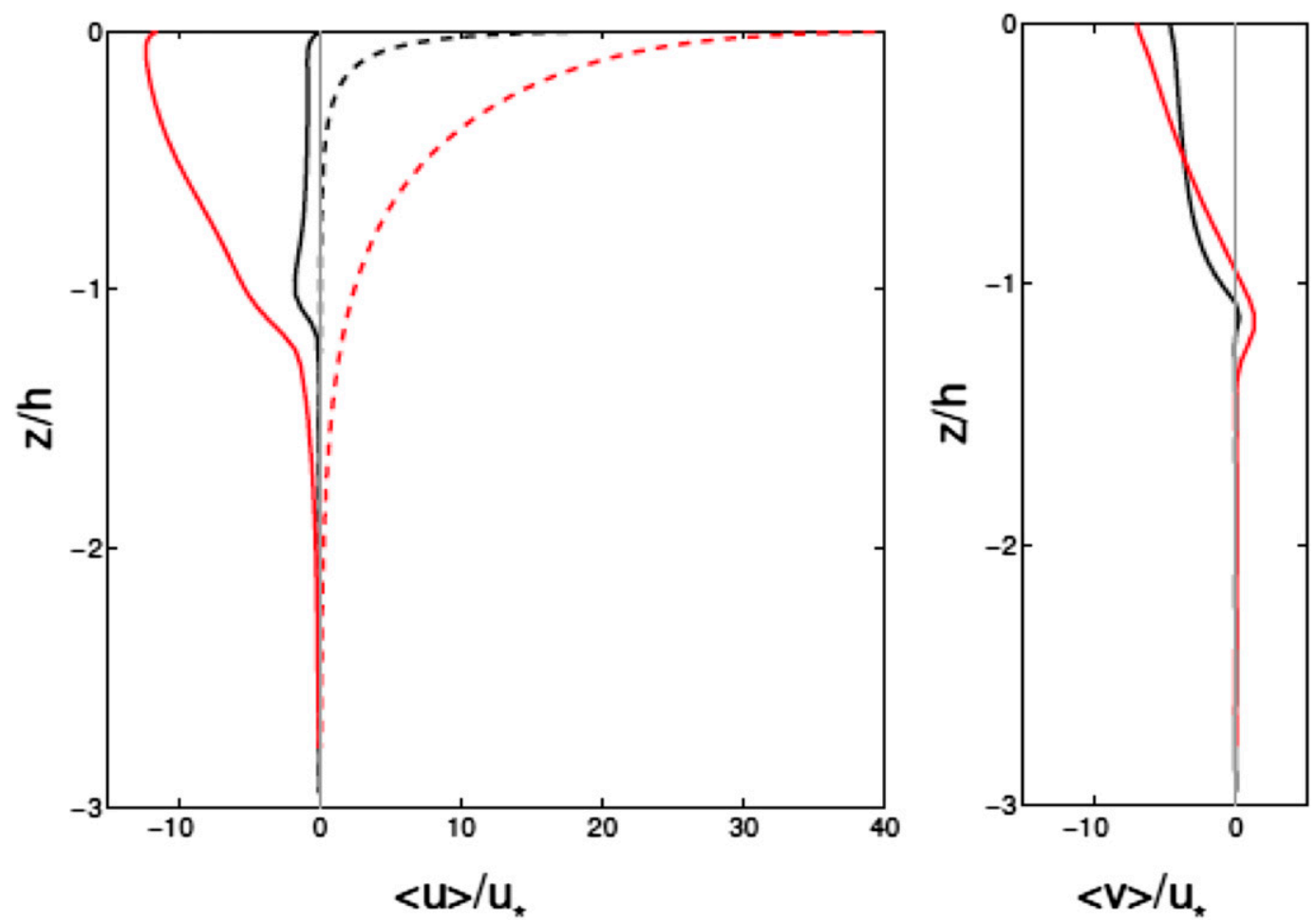

FIG. 2. (left) Eastward and (right) northward velocities for two cases in the case set Us1: wind-wave equilibrium (black) and with an added swell with $U^{s}=0.25 \mathrm{~m} \mathrm{~s}^{-1}, D^{s}=16 \mathrm{~m}$, and a wind-aligned $\theta^{s}=0$ (red). The solid lines are the mean velocity, and the dashed lines are the Stokes drift $\mathbf{u}_{\mathrm{st}}$.

The solutions (7) and (8) have a left-right symmetry with respect to the swell direction $\theta^{s}$ relative to the wind direction (here E). Define $\hat{\mathcal{U}}$ as the solution without swell (i.e., for $U^{s}=0$ ). Then for $U^{s} \neq 0$ and general $\theta^{s}$, the difference fields $\mathcal{V}_{\theta^{s}}=\mathcal{U}_{\theta^{s}}-\hat{\mathcal{U}}$ are equivalent when rotated by the horizontal angle $2 \theta^{s}$; that is,

$$
\mathcal{V}_{\theta^{s}}=\exp \left[i 2 \theta^{s}\right] \mathcal{V}_{-\theta^{s}}
$$

The same rotational symmetry holds for $-\kappa_{o} \partial_{z} \mathcal{U}$, which is the Ekman-Stokes equivalent of Reynolds stress minus the upward-integrated breaker forcing [i.e., $\mathbf{R}(z)$ in (15)].

For flows impulsively started from rest, the preceding steady solution $\mathcal{U}(z)$ is augmented by an inertial oscillation $\mathcal{U}_{\text {in }}(z, t)$ such that their sum is zero at $t=0$ at all depths. With an additional acceleration term $\partial_{t} \mathcal{U}$ in (5), the homogeneous solutions are the eigenmodes

$$
\mathcal{U}_{\text {in }} \propto \exp \left[-i f t-\kappa_{o} k^{2} t\right] \cos [k z], \quad k \geq 0,
$$

which imply vertical oscillation with wavenumber $k$, anticyclonic temporal rotation of the current direction at an inertial frequency $f$, and amplitude decay at a rate $\kappa_{o} k^{2}$. The longer-lasting components have smaller $k$, and hence a larger vertical scale. So an estimate of the initial inertial current amplitude is made with the steady velocity averaged over the boundary layer, that is, the transport magnitude divided by the Ekman depth scale

$$
U_{\text {in } 0}=\frac{1}{h_{e}}\left|\int_{-\infty}^{0} \mathcal{U} d z\right|,
$$

using (9) to evaluate the integral.

For comparison with the LES results below, the Ekman-Stokes solution is normalized by $u *$ for velocity, $h_{e}$ for depth, and $u_{*}^{2}$ for the equivalent of Reynolds stress (i.e., $-\kappa_{o} \partial_{z} U$ or $-\kappa_{o} \partial_{z} \mathbf{u}$ ). As will be shown, the matches of the Ekman-Stokes solutions to the LES results are not precise in their vertical profile structure, as is to be expected with the strong simplifications of uniform density and eddy viscosity (cf. Fig. 6, described in greater detail below) and a monochromatic wind-sea profile for $\mathbf{u}_{\mathrm{st}}^{w}(z)$ (cf. Fig. 2). The Ekman-Stokes parameters are chosen, first, by exactly matching $u_{*}, U^{s}, \theta^{s}$, and $D^{s}$ with their LES values. Second, the measured LES boundary layer depth $h$ (which is limited by stable stratification rather than $\sqrt{2 \kappa_{o} / f}$ for $h_{e}$ ) is used for its normalizations of $z$ and Reynolds stress. Third, for the remaining Ekman-Stokes parameters- $U^{w}, D^{w}$, and $\kappa_{o}$ - the simple profile assumptions for the wind-sea Stokes drift velocity and diagnosed 

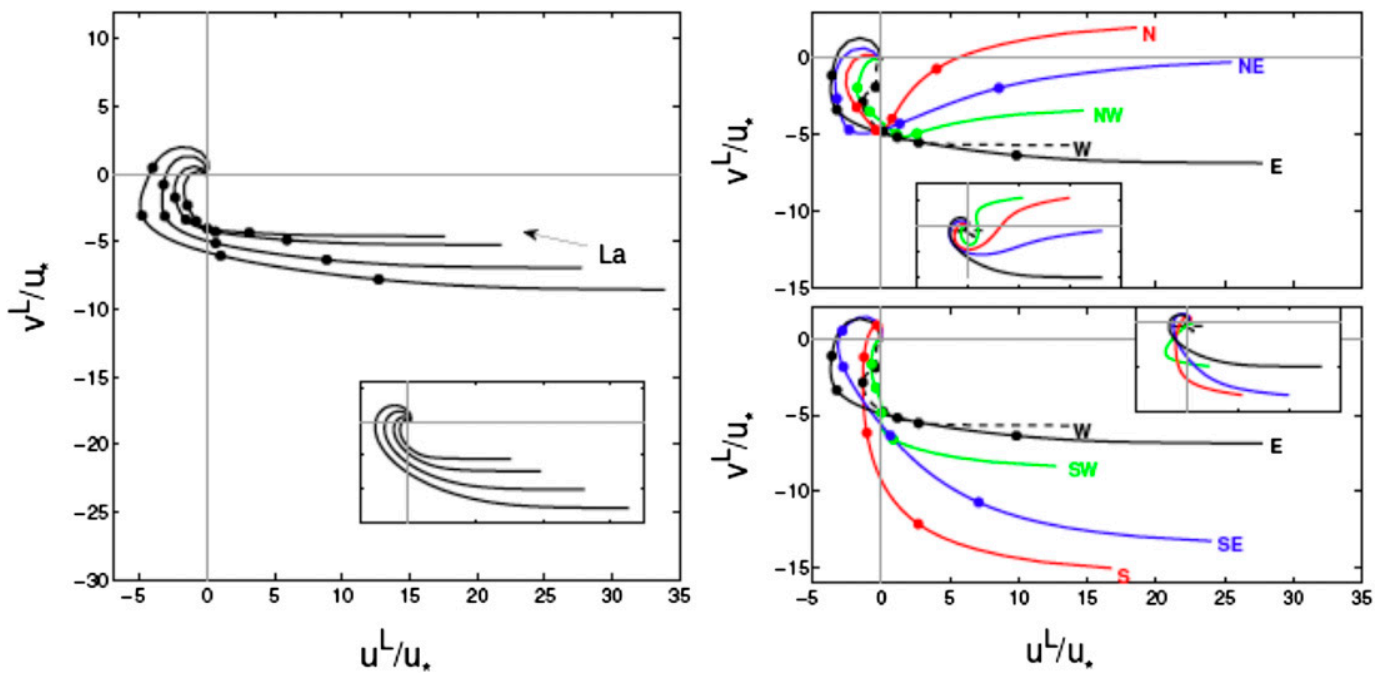

FIG. 3. Hodographs of Lagrangian-mean velocity, $\mathbf{u}^{L}(z)$ in (13), for the case sets (left) Us1 and (right) $\Theta s$. The dots along the spirals indicate depths of $-z / h=(0.1,0.3,0.6,0.9)$. The arrow indicates increasing La from the largest (outer spiral) to no swell (inner spiral) (left). The $\theta^{s}$ values are labeled by their compass directions, and, for clarity, the $\theta^{s} \geq 0$ are separated from the $\theta^{s} \leq 0$ cases (right). The insets show the equivalent spirals from the Ekman-Stokes model, with the $\theta^{s}= \pm \pi(\mathrm{W})$ profile having very small $\mathbf{u}^{L}(z)$ values at all depths.

eddy viscosity do not correspond to the more complex profiles in the LES solution. Therefore, the EkmanStokes parameter values are chosen-0.125 $\mathrm{m} \mathrm{s}^{-1}, 1.25 \mathrm{~m}$, and $0.025 \mathrm{~m}^{2} \mathrm{~s}^{-1}$, respectively-to give the best visual comparisons below in Figs. 3-5. These parameter values are not ill matched to the more complex $\mathbf{u}_{\mathrm{st}}^{w}(z)$ and $\kappa(z)$ profiles in Figs. 2 and 6, respectively. Furthermore, the normalized Ekman-Stokes profile shapes are not highly sensitive to these parameter choices. At best, the EkmanStokes model is a simple explanation for some of the $\mathbf{u}_{\mathrm{st}}^{s}$ dependencies exhibited in the more complete LES solutions, and by this standard it proves to be useful.

\section{Mean current and Reynolds stress}

From a larger-scale perspective, the most important outcomes for the boundary layer are the quasi-steady mean horizontal current and slowly eroding density stratification after an initial spinup period of about a day. After filtering out the inertial current that arises from the
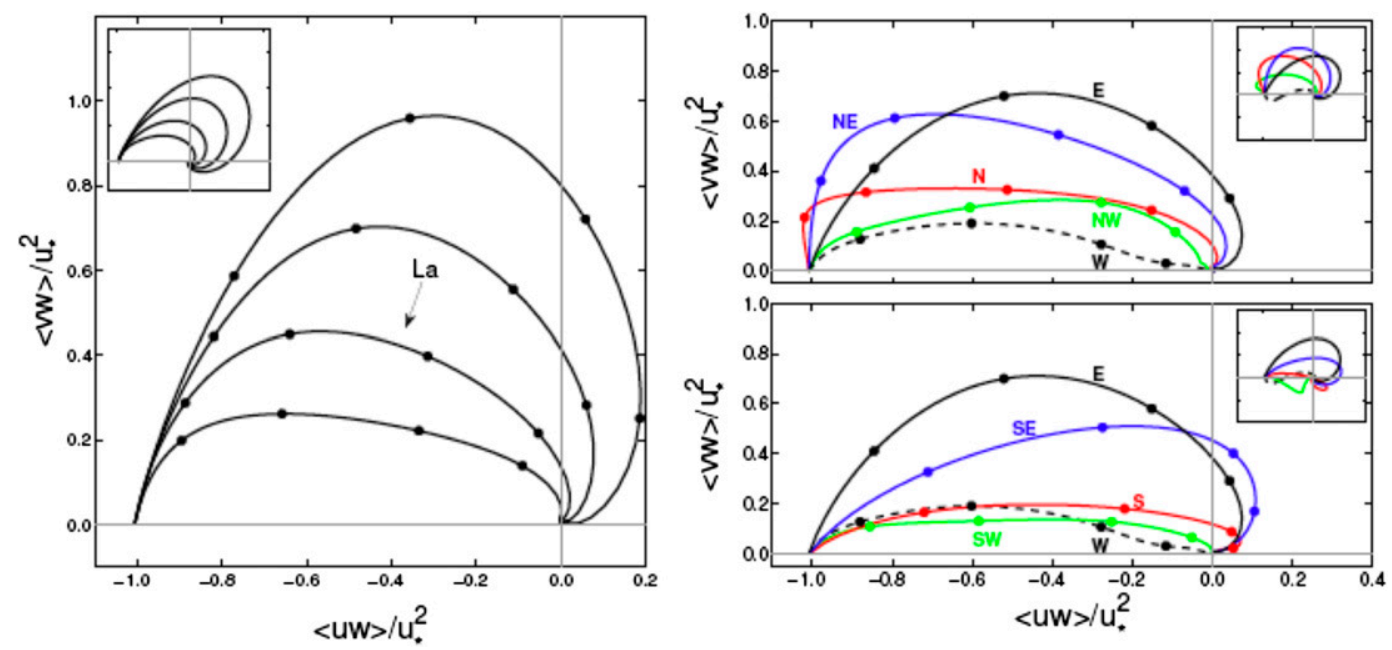

FIG. 4. Hodographs of the Reynolds stress plus breaker impulse, $\mathbf{R}(z)$ in (15), for the case sets (left) Us1 and (right) $\Theta$ s. The format is the same as in Fig. 3. For the insets, the comparable quantity in the Ekman-Stokes model is $-\kappa_{o} \partial_{s} \mathbf{u}(z)$ : $\mathbf{R}(0)=-u_{*}^{2}$ at the surface, and $\mathbf{R} \rightarrow 0$ at depth. 

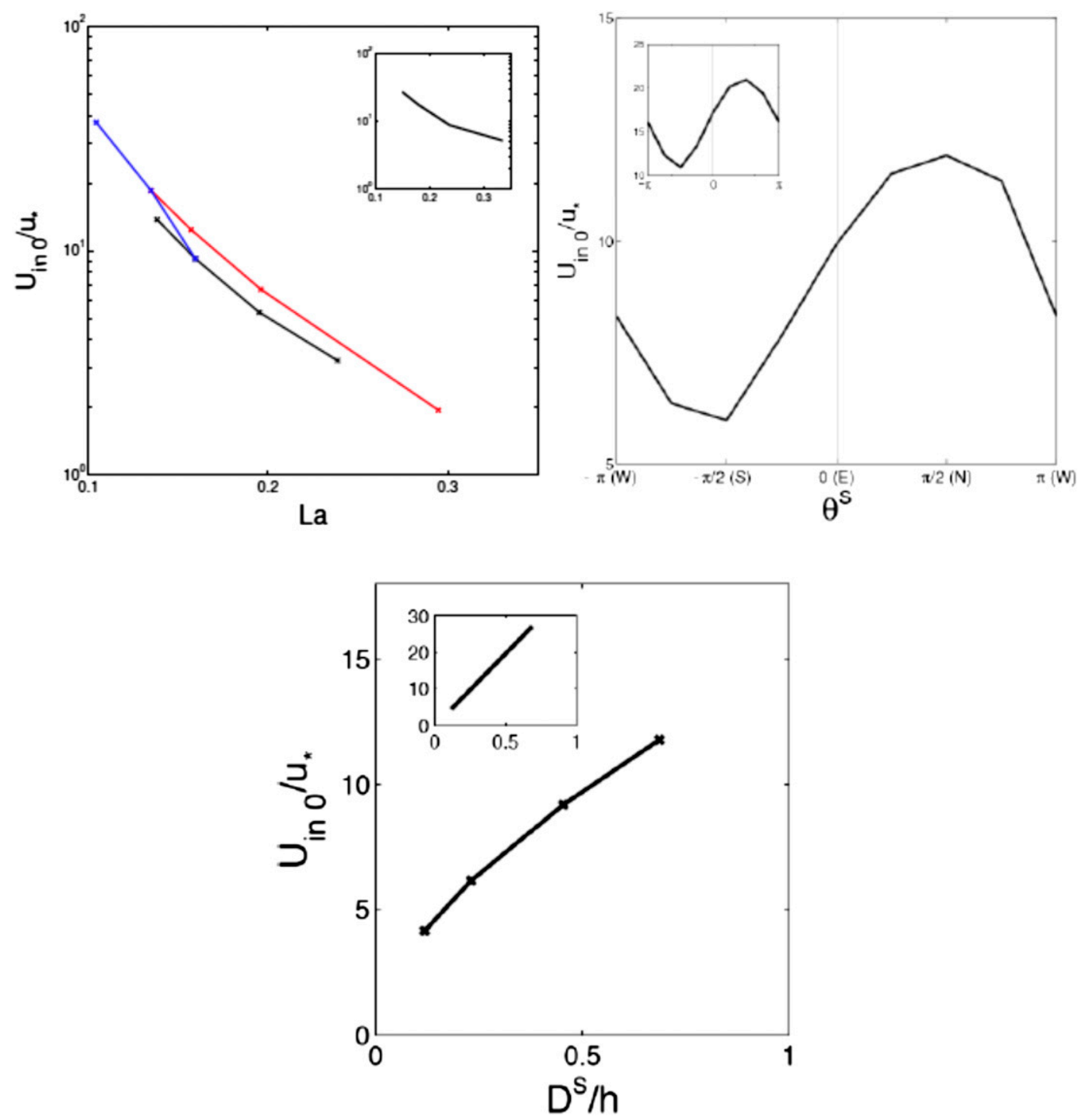

FIG. 5. Inertial current amplitude $U_{\text {in } 0}$ averaged over the boundary layer ( $\left.-h \leq z \leq 0\right)$ as a function of (top, left) La for case sets Us1 (black), Us2 (red), and Ua (blue); as a function of (top, right) $\theta^{s}$ for case set $\Theta$ s; and as a function of (bottom) $D^{s}$ for case set Ds. The insets show the equivalent results from the Ekman-Stokes model using (12).

impulsive start to the boundary layer flow, the mean current profile $\langle\mathbf{u}\rangle(z)$ in Fig. 2 is a combination of a clockwiserotating Ekman spiral in $\mathbf{u}^{L}$ and an anti-Stokes Eulerian flow with $\langle\mathbf{u}\rangle$ opposing $\mathbf{u}_{\text {st }}$, as in the Ekman-Stokes solution (7). Both features are familiar attributes of equilibrium Langmuir turbulence (e.g., McWilliams et al. 1997); for example, if $\mathbf{u}_{\mathrm{st}}=0$ (as in case NB defined in Table 1; not shown), the Eulerian $\langle u\rangle>0$ near the surface because there is no anti-Stokes effect. With an added swell aligned with the wind, the Stokes drift velocity is stronger and deeper. The wind-aligned mean velocity is increasingly upwind, the crosswind velocity is more nearly linear in its profile and reverses near the boundary layer base, and both components extend somewhat deeper because $h$ is a bit larger through enhanced entrainment (section 6). Although such large swell has not been examined before, these behaviors are not surprising for Langmuir turbulence solutions.

The anti-Stokes effect is partly canceled in the Lagrangian-mean flow profile

$$
\mathbf{u}^{L}(z)=\langle\mathbf{u}\rangle+\mathbf{u}_{\mathrm{st}} .
$$

In the Ekman-Stokes model the cancellation is complete in the transport (9), as is also true in the LES that satisfies the same transport relation (16). When the swell amplitude $U^{s}$ increases for wind-aligned swell (i.e., $\theta^{s}=0$; Fig. 3, left), the surface maximum in $\mathbf{u}^{L}$ is larger, and the Ekman spiral is fatter; that is, the flow in the bulk of 

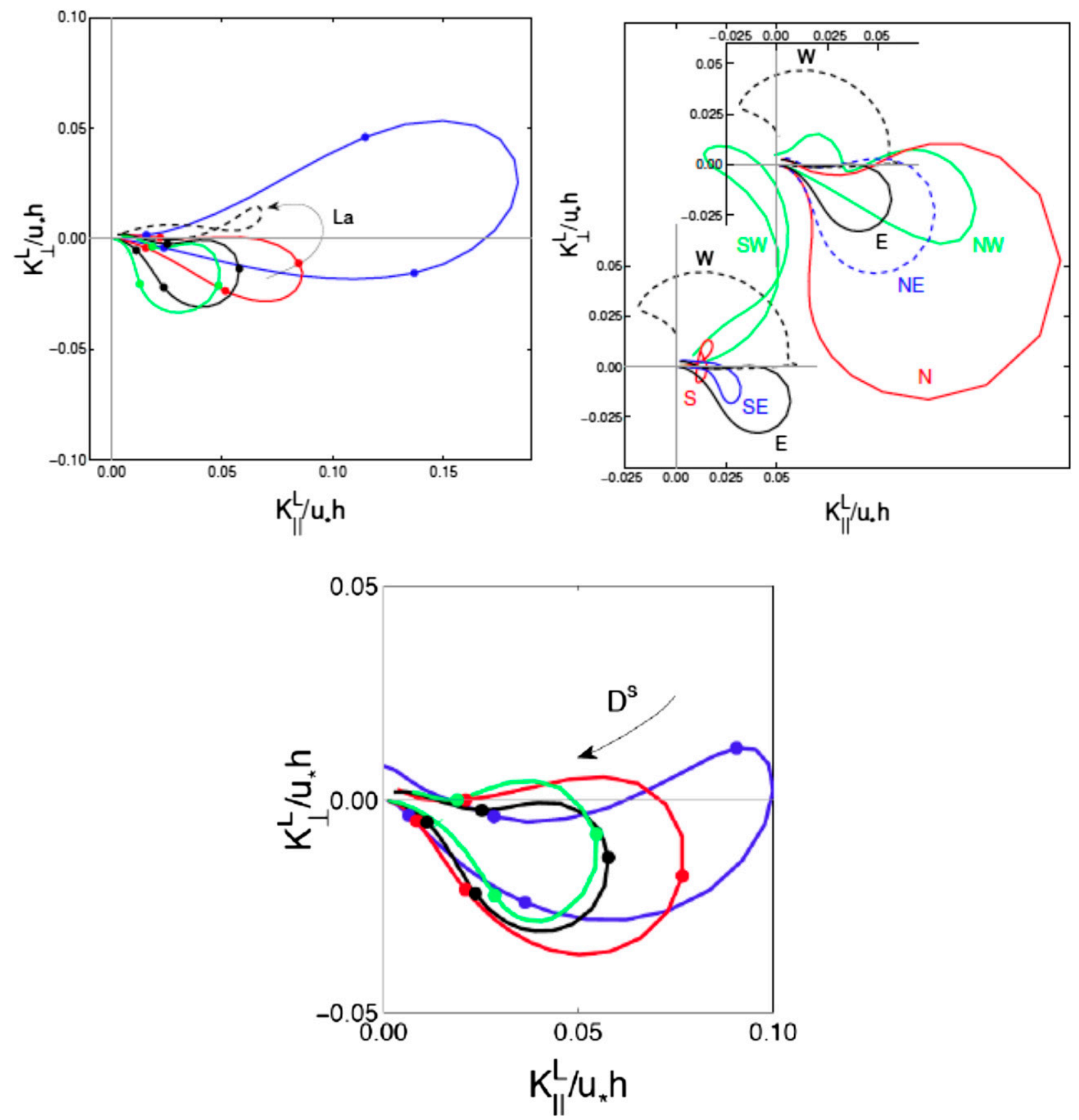

FIG. 6. Hodographs of the eddy viscosity vector, $\boldsymbol{\kappa}^{L}=\left(\kappa_{\|}^{L}, \kappa_{\perp}^{L}\right)$ in (18) normalized by $u * h$, for the case sets (left) Us1, (right) $\Theta \mathrm{s}$, and (bottom) Ds. The format is similar to Fig. 3. (top, left) The cases are for $U^{s}=0$ (blue), 0.1 (red), 0.25 (black), and $0.4 \mathrm{~m} \mathrm{~s}^{-1}$ (green), plus the case NB with $\mathbf{u}_{\text {st }}=0$ (defined in Table 1; black dashed); the curved arrow indicates the direction of increasing La. The loops are traversed counterclockwise with depth from the origin out to a middepth max and back to the origin. The Ekman-Stokes model (section 3 ) would be represented as a point on the abscissa at $\left(\kappa_{o}, 0\right) / u_{*} h_{e}$, and the standard K-profile parameterization (KPP) scheme (Large et al. 1994) would be a line traversed by $(K(z), 0) / u_{*} h$, with $K(z)$ a convex positive function of $z$ that starts and ends near the origin.

the boundary layer is stronger. Compared to case NB without Stokes drift (defined in Table 1; not shown), these wave-influenced cases have a stronger surface $\mathbf{u}^{L}$ and a fatter Ekman. The same qualitative trend with $\mathrm{La}$ occurs in the Ekman-Stokes solution for analogous parameter values (section 3), which is plotted in the inset. When the swell direction $\theta^{s}$ varies away from wind alignment to the east (Fig. 3, right), $\mathbf{u}^{L}$ at the surface weakens and rotates toward the swell, but only over a limited azimuthal range. In the interior of the boundary 
layer, the upwind velocity component is strongest-the Ekman spiral is fattest-in the $\theta^{s}$ range from southeast (SE) to northeast (NE) and is much weaker over the rest of the compass range. Again, these behaviors are similar to those in the Ekman-Stokes model (shown as insets in Figs. 3, 4, and 5). In Fig. 3, there is a rough left-right symmetry with a sign reversal in $\theta^{s}$ [e.g., north $(\mathrm{N})$ versus south (S)]. The dependency of $\mathbf{u}^{L}(z)$ on the swell depth scale $D^{s}$ (i.e., in the case set Ds) is rather slight and is not shown; with a smaller $D^{s}, \mathbf{u}^{L}(z)$ is slightly more surface intensified. Of course, the anti-Stokes aspect in $\langle u\rangle$ is larger and reaches deeper with larger $D^{s}$, as in (7).

The mean horizontal momentum balance (averaged over the inertial oscillation) is

$$
f \hat{\mathbf{z}} \times\left(\langle\mathbf{u}\rangle+\mathbf{u}_{\mathrm{st}}\right)=-\partial_{z}\left\langle\mathbf{u}^{\prime} w^{\prime}\right\rangle+\hat{\mathbf{x}} A,
$$

with a surface boundary condition of $\left\langle\mathbf{u}^{\prime} w^{\prime}\right\rangle(0)=0$ due to the replacement of wind stress by the mean breaker momentum impulse $\mathbf{A}(z)>0$ in a thin layer near the surface. The velocity on the left side is the Lagrangianmean flow $\mathbf{u}^{L}(z)$, and one sees the constraint that an anti-Stokes Eulerian flow $\langle\mathbf{u}\rangle$ develops with an increasing Stokes drift $\mathbf{u}_{\text {st }}$ and fixed momentum forcing by the wind, with the Reynolds stress profile unlikely to exceed the surface stress. The right side forcing in (14) is minus the divergence of the difference between the Reynolds stress and the upward integral of the breaker impulse:

$$
\mathbf{R}(z)=\left\langle\mathbf{u}^{\prime} w^{\prime}\right\rangle-\hat{\mathbf{x}} \int_{-H}^{\mathbf{z}} A d z^{\prime}
$$

Because of the boundary condition on Reynolds stress and the normalization for $A$, this has a surface value of $\mathbf{R}(0)=-u_{*}^{2}$. Thus, the transport integral,

$$
\int_{-H}^{0} \mathbf{u}^{L} d z=-\frac{1}{f} \hat{\mathbf{z}} \times \hat{\mathbf{x}} \int_{-H}^{0} A\left(z^{\prime}\right) d z^{\prime}=-\hat{\mathbf{y}} \frac{u_{*}^{2}}{f},
$$

is independent of the swell amplitude [cf. (9)], despite its evident influence on the profile shape of $\mathbf{u}^{L}(z)$ (Fig. 3).

The Reynolds stress hodograph for $\mathbf{R}(z)$ in Fig. 4 becomes much fatter when $U^{s}$ is large and $\theta^{s}$ is in the same quadrant as the wind direction. With large enough $U^{s}$ (which occurs in the case set Us2, not shown), the magnitude of the crosswind $\left\langle v^{\prime} w^{\prime}\right\rangle$ even exceeds the surface wind stress. For swell rotated to the left of the wind $\left(\theta^{s}>0\right)$, the $\mathbf{R}(z)$ hodograph tilts to the left and vice versa for $\theta^{s}<0$; but again, the range in directional tilt is much less than the range in $\theta^{s}$. For the quadrants away from the wind [from south to north through west (W)], the hodograph becomes quite thin. These dependencies on $U^{s}$ and $\theta^{s}$ are also present in the Ekman-Stokes model (insets), although the shapes of its hodographs are not the same, and the left-right symmetry in $\theta^{s}$ is not as visually apparent, in spite of the fact that it does have the particular $\theta^{s}$ symmetry (10). Again, the $D^{s}$ dependencies of the Reynolds stress (not shown) are much smaller than those for $U^{s}$ and $\theta^{s}$.

The mean momentum balance (14) may equivalently be expressed in terms of a Lagrangian eddy viscosity tensor defined by

$$
-\left\langle\mathbf{u}^{\prime} w^{\prime}\right\rangle=\kappa^{L} \mathbf{R} \cdot \partial_{z} \mathbf{u}^{L}, \quad \mathbf{R}=\left(\begin{array}{cc}
\cos \theta^{L} & -\sin \theta^{L} \\
\sin \theta^{L} & \cos \theta^{L}
\end{array}\right),
$$

where $\mathbf{R}(\theta)$ is the horizontal rotation matrix representing the rotation of the shear direction into the opposite of the Reynolds stress direction. Here, $\kappa^{L}(z)$ is the positive scalar magnitude, and $\theta^{L}(z)$ is the rotation angle. A conventional eddy viscosity model has $\theta^{L} \equiv 0$, but this is not sufficient to characterize an Ekman layer with surface waves (McWilliams et al. 2012, section 3d). Alternatively, an eddy viscosity vector is defined in terms of components of the Reynolds stress parallel and perpendicular to the mean Lagrangian shear:

$$
\begin{aligned}
& \kappa_{\|}^{L}(z)=\kappa^{L} \cos \left[\theta^{L}\right]=-\frac{\partial_{z} \mathbf{u}^{L} \cdot\left\langle\mathbf{u}^{\prime} w^{\prime}\right\rangle}{\left(\partial_{z} \mathbf{u}^{L}\right)^{2}}, \\
& \kappa_{\perp}^{L}(z)=\kappa^{L} \sin \left[\theta^{L}\right]=-\hat{\mathbf{z}} \cdot \frac{\partial_{z} \mathbf{u}^{L} \times\left\langle\mathbf{u}^{\prime} w^{\prime}\right\rangle}{\left(\partial_{z} \mathbf{u}^{L}\right)^{2}} .
\end{aligned}
$$

In the conventional model, $\kappa_{\|}^{L}>0$ and $\kappa_{\perp}^{L}=0$.

In McWilliams et al. (2012) it is shown that a Lagrangian eddy viscosity representation has simpler vertical structure in $\kappa(z)$ and a smaller range of $\theta(z)$ variation than a conventional Eulerian one (i.e., defined with $\mathbf{u}^{L}$ replaced by $\langle\mathbf{u}\rangle$ in the preceding formulas) for unstratified, wind-wave equilibrium Langmuir turbulence, especially in the near-surface region where $\mathbf{u}_{\mathrm{st}}$ is not small. The same is true in the present stratified cases with swell-waves. ${ }^{2}$ The hodograph of the eddy viscosity vector $\boldsymbol{\kappa}^{L}(z)=\left(\kappa_{\|}^{L}(z), \kappa_{\perp}^{L}(z)\right)$ (where the parentheses are standard math notation for expressing components of a vector in this type of serial form) in Fig. 6 (left) shows both the increase in the magnitude of $\kappa^{L}$ in a wind-sea compared to the absence of waves (case NB; defined in Table 1) and the ensuing decrease with added swell,

\footnotetext{
${ }^{2}$ Similarly, Harcourt (2013) proposes a local second-moment turbulence closure model based on Lagrangian-mean shear rather than Eulerian.
} 
$U^{s} \neq 0$. This nonmonotonic dependence on La is somewhat surprising, and this is further discussed at the end of this section. The vertical profile of $\kappa^{L}(z)$ has a middepth maximum and vanishes at the edges of the boundary layer in all cases (i.e., the looping curves in the hodograph that start and end at the origin). Without waves the loop is very thin, indicating that $\theta^{L} \approx 0$, but it is not thin for either a wind-sea alone or a wind-sea with added swell. The wind-sea case with $U^{s}=0$ has $\theta_{\kappa}^{L}<0$ in the Stokes layer near the surface and $\theta_{\kappa}^{L}>0$ deeper in the interior of the boundary layer; the same structure occurs in the unstratified, wavy Ekman layer (McWilliams et al. 2012). With increasing $U^{s}$, the loops shrink and rotate clockwise in $\theta^{L}$, so that $\theta^{L}(z)<0$ at almost all depths with the largest $U^{s}$. With variable $\theta^{s}$ (Fig. 6 , right), the magnitude of $\boldsymbol{\kappa}^{L}$ increases with leftward swell rotation up to $\theta^{s}=\pi / 2$ (north), then begins to shrink again and $\theta^{L}$ rotates to the left. With rightward rotation in $\theta^{s}$, the magnitude decreases to a minimum near $\theta^{s}=-\pi / 2$ (south), and $\boldsymbol{\kappa}^{L}$ assumes a distended loop shape for $\theta^{s}=-3 \pi / 2$ [southwest (SW)]. This is a very substantial left-right $\theta^{s}$ asymmetry in the boundary layer response to swell.

The Ekman-Stokes model (section 3) is not apt for these eddy viscosity behaviors because $\left(\kappa_{o}, 0\right)$ is just a point on the positive abscissa of these hodographs. This implies that the analytic model is not reliable for the detailed profile structure of the mean flow and Reynolds stress, even if it is useful for indicating their qualitative dependencies on the swell-wave parameters. In contrast, a "Lagrangian diffusion" Ekman-Stokes model-analogous to (5) and its boundary conditions but with the Eulerian diffusive flux $\kappa_{o} \partial_{z} \mathcal{U}$ replaced by a Lagrangian flux $\kappa_{o}^{L} \partial_{z} \mathcal{U}^{L}$-would be isomorphic to a standard Ekman model without waves; that is, it would show no dependencies on the added swell and thus have no explanatory value for Figs. 3 and 4, as well as being only a point located at $\left(\kappa_{o}^{L}, 0\right)$ in Fig. 6 .

Finally, there is an appreciable dependency of $\kappa^{L}(z)$ on $D^{s}$ in the case set Ds (Fig. 6, bottom), with a larger eddy viscosity magnitude as $D^{s}$ is decreased, while maintaining a roughly similar loop shape as in Fig. 6 (left).

The complexity of the $\boldsymbol{\kappa}^{L}(z)$ profiles and their variations with swell-wave parameters in Fig. 6 are challenging for achieving an accurate boundary layer parameterization for the shape of $\langle\mathbf{u}\rangle(z)$. On the other hand, there is evident skill shown in the preceding figure insets for the very simple parameterization used in the Ekman-Stokes model with a constant $\kappa_{o}$ (section 3). The implications of this trade-off between accuracy and simplicity are further discussed in section 8 .

It is known from previous work (McWilliams et al. 1997; McWilliams and Sullivan 2000) that the eddy viscosity magnitude increases due to the presence of waves compared to a shear boundary layer without them (e.g., case NB; defined in Table 1). This is a different trend than the present result that $\kappa^{L}$ decreases with the addition of an increasing swell-wave amplitude to an equilibrium wind-wave field. The viscosity magnitude from (17) is the ratio of Reynolds stress and Lagrangianmean shear magnitudes

$$
\kappa^{L}=\frac{\left|\left\langle\mathbf{u}^{\prime} w^{\prime}\right\rangle\right|}{\left|\partial_{z} \mathbf{u}^{L}\right|}
$$

while the mean horizontal momentum balance (14) below the wave-breaking layer implies

$$
\left|\partial_{z} \mathbf{u}^{L}\right| \approx \frac{1}{f}\left|\partial_{z}^{2}\left\langle\mathbf{u}^{\prime} w^{\prime}\right\rangle\right|
$$

Thus,

$$
\kappa^{L} \approx f \frac{\left|\left\langle\mathbf{u}^{\prime} w^{\prime}\right\rangle\right|}{\left|\partial_{z}^{2}\left\langle\mathbf{u}^{\prime} w^{\prime}\right\rangle\right|} .
$$

To explain the trend in $\kappa^{L}$ with decreasing La, for example, for wind-aligned waves, one can examine the trends in the numerators and denominators of (19) or (21). Both $\left|\left\langle\mathbf{u}^{\prime} w^{\prime}\right\rangle\right|$ and $\left|\left\langle\partial_{z} \mathbf{u}^{L}\right\rangle\right|$, hence $\left|\partial_{z}^{2}\left\langle\mathbf{u}^{\prime} w^{\prime}\right\rangle\right|$ as well, are decreasing functions of depth, while their ratio $\kappa^{L}$ has a convex shape with a maximum near the middle of the boundary layer (Fig. 6). With decreasing La, the $\left\langle\mathbf{u}^{\prime} w^{\prime}\right\rangle(z)$ profile shows a systematically increasing middepth numerator. The denominator $\left|\left\langle\partial_{z} \mathbf{u}^{L}\right\rangle\right|$ first decreases with the presence of weak waves, then around $\mathrm{La} \approx 1$ begins to increase with stronger waves, ${ }^{3}$ thus implying the reversing trends in $\kappa^{L}$. Consistent with (20), the Reynolds stress profile curvature (i.e., its second derivative with depth) first diminishes with decreasing $\mathrm{La}$, then because of the tendency of the hodograph to fatten (Fig. 4) with added swell, it also increases. The Ekman-Stokes model with its fixed Eulerian $\kappa_{o}$, in contrast, has monotonically increasing numerators and denominators, and their middepth ratios as La decreases (not shown). The implication is that, in the LES solution, changes in the wave field change both the shape of the Langmuir circulations (section 7) with their significant contribution to the momentum flux profile (e.g., demonstrated in McWilliams et al. 2012) and the outcome of the anti-Stokes competition in the Lagrangian

\footnotetext{
${ }^{3}$ This result is based on LES solutions with an equilibrium windwave Stokes profiles whose amplitude is artificially reduced to increase La above its equilibrium value of 0.3 . These solutions are not shown here explicitly, but both Harcourt and D'Asaro (2008) and Grant and Belcher (2009) show results for this larger La regime.
} 
$\mathbf{u}^{L}(z)$ profile in seemingly subtle ways that lead to the diagnosed $\kappa^{L}(z)$ changes with La. A more simplistic argument is that the numerator is plausibly constrained by the wind stress magnitude $u_{*}^{2}$, while the denominators are freer to grow with increasing $\partial_{z} \mathbf{u}_{\mathrm{st}}$; however, this argument does not faithfully capture the decreasing $\left|\partial_{z} \mathbf{u}^{L}\right|$ in the larger La regime.

\section{Inertial current}

As mentioned, the horizontally averaged current exhibits inertial currents generated in the initialization shock. After adjustment, their vertical structure is approximately uniform in $z$ over the boundary layer and weak below. For each LES case, a depth-averaged inertial amplitude $U_{\text {in } 0}$ is fit during the analysis period of one inertial period. The dependencies with $U^{s}$ and $\theta^{s}$ are shown in Fig. 5. The inertial amplitude increases with $U^{s}$ (decreasing La), and the three case sets with variable $\mathrm{La}$ (Us1, Us2, and Ua) all show a similar functional shape. The $\theta^{s}$ variations have an inertial current amplitude dependency with a roughly $a+b \sin$ $\left[\theta^{s}\right]$ dependency. These behaviors are qualitatively similar in the Ekman-Stokes (12), as is evident by comparison with the insets in Fig. 5. The $U_{\text {in } 0}$ also increases approximately linearly with $D^{s}$ in the LES (Fig. 5, bottom), consistent with the prediction in (12); that is,

$$
\frac{U_{\text {in } 0}}{u_{*}}=\frac{1}{u_{*} h}\left|\frac{i \mathcal{T}}{f}-D^{w} U^{w}-D^{s} U^{s} \exp \left[i \theta^{s}\right]\right| \approx \frac{D^{s} U^{s}}{u_{*} h}
$$

for large $D^{s}$ and $U^{s}$. This relation explains the increase in $U_{\text {in } 0}$ with decreasing $\mathrm{La}$; that is, as $D^{s}$ and $U^{s}$ increase, $\mathrm{La}$ decreases. It also explains the dependency on $\theta^{s}$. When $\theta^{s}$ is in the NE quadrant, with the minus sign in (22) the magnitude of $U_{\text {in } 0}$ increases in the first relation in (22) by the swell contribution being in between the southward contribution from wind stress and the westward negative Stokes drift contribution from the wind-sea. Conversely, when $\theta^{s}$ is in the SW quadrant, the swell effect subtracts from the contributions from stress and wind-sea, making $U_{\text {in } 0}$ smaller. Overall, large swell amplifies the inertial response to a sudden wind change. ${ }^{4}$ Furthermore, compared to case NB without Stokes drift (defined in Table 1; not shown), these wave-influenced cases have a stronger inertial current response.

\footnotetext{
${ }^{4}$ In LES cases that go beyond the largest $D^{s}$ value in case set Ds, the further increases in $D^{s}$ lead to little change in the turbulent intensity and fluxes. However, the anti-Stokes component in $\langle u\rangle(z)$ continues to deepen proportionally, and the amplitude of the inertial response increases. No evident local instability below the boundary layer arises in association with the deep $\langle u\rangle(z)$ shear.
}

\section{Turbulent intensity, entrainment rate, and energy production}

Define the turbulent kinetic energy profile as

$$
e(z)=\frac{1}{2}\left(\left\langle\mathbf{u}^{2}\right\rangle+\left\langle w^{2}\right\rangle\right)
$$

The prime indicates the subtraction of the horizontally averaged flow at each time, that is, excluding both the mean and inertial currents. The $e$ is the isotropic measure of turbulent intensity, and the vertical velocity variance $\left\langle w^{2}\right\rangle$ is useful to compare to float measurements in the surface boundary layer (Harcourt and D'Asaro 2008). These two profile quantities are plotted in Fig. 7. They show a surface-intensified $e(z)$ and a shallow maximum in $\left\langle w^{2}\right\rangle(z)$. With wind-aligned swell, both $e$ and $\left\langle w^{2}\right\rangle$ increase compared to the wind-sea case; this is similar to their increase in equilibrium Langmuir turbulence compared to a boundary layer without the Stokes vortex force (case NB defined in Table 1; not shown). Profiles are also shown for $\theta^{s}= \pm \pi / 2$. For northward swell, $e$ is as large as with wind alignment, whereas for southward swell $e$ is much reduced. Both $e(z)$ and $\left\langle w^{2}\right\rangle(z)$ show some oscillatory complexity in the northward case, including elevated variances in the deep interior below the boundary layer (i.e., $z<-h$ ) that indicate increased internal gravity wave excitation by the boundary layer turbulence impinging on the top of the pycnocline (Polton et al. 2008).

This is another asymmetric response to the swell angle $\theta^{s}$, and it is in the same sense as the stronger inertial current response for $\theta^{s}>0$ (section 5). However, there is no temporal correlation of turbulent intensity with the phase of the inertial current. This implies that the enhanced vertical shear at the base of the boundary layer when the inertial current aligns with the mean current shear is not an important source of turbulent energy production [in contrast to the situation of "inertial resonance" when the wind rotates in phase with the inertial current (Price 1981)].

To demonstrate the swell-wave parameter dependencies, Fig. 8 shows $e$ and $\left\langle w^{2}\right\rangle$ after averaging both within the boundary layer and in the interior layer below. The turbulent intensities increase with smaller La in both layers, with the one exception of boundary layer $\left\langle w^{2}\right\rangle$ for the most extreme case of weak winds in case set Ua. Through an argument that relates turbulent intensity to the efficacy of Stokes production (i.e., $\mathcal{P}_{\text {st }}=-\left\langle\mathbf{u}^{\prime} w^{\prime}\right\rangle \cdot \partial_{z} \mathbf{u}_{\text {st }}>0$ ) in the turbulent kinetic energy balance (Harcourt and D'Asaro 2008; Grant and Belcher 2009), the scaling prediction is $e,\left\langle w^{2}\right\rangle \sim$ $\mathrm{La}^{-4 / 3}$. This comparison curve is also drawn, and it is 

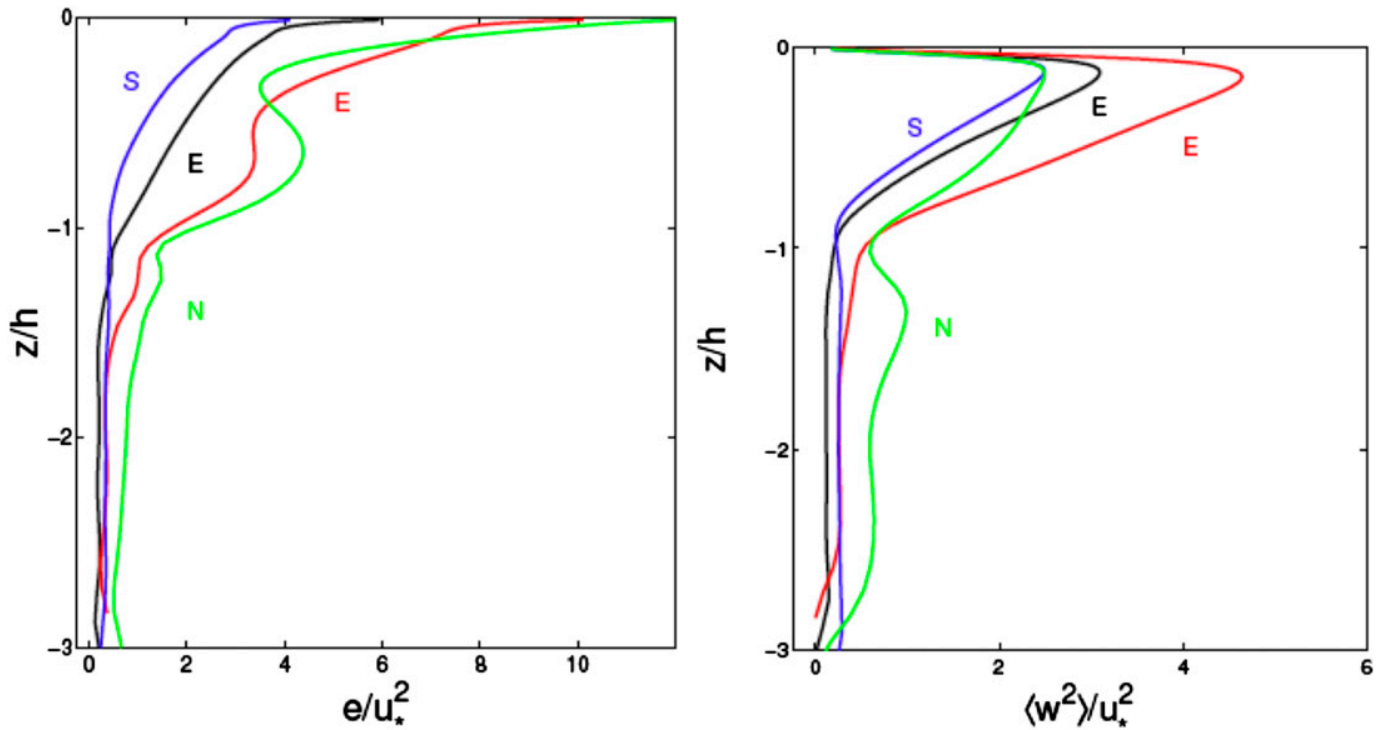

FIG. 7. Turbulent variance profiles: (left) $e(z)$ and (right) $\left\langle w^{2}\right\rangle(z)$ for the case sets Us1 with $U^{s}=0$ (black) and $\Theta \mathrm{s}$ with $U^{s}=0.25 \mathrm{~m} \mathrm{~s}^{-1}$ and $\theta^{s}$ oriented toward east (red), north (green), and south (blue).

roughly consistent with the La dependencies in the boundary layer for these cases with added swell that extend to smaller La than previously explored. The increases in internal-wave variance below the boundary layer are an even steeper function of inverse La, roughly $\sim \mathrm{La}^{-2}$.
The swell-wave direction dependencies show the greatest inertial current and turbulent intensity for $\theta^{s}$ in the NE quadrant, consistent with Figs. 5 and 7. The $\theta^{s}$ dependency in Fig. 8 is a roughly symmetric decrease away from wind alignment for $\left\langle w^{2}\right\rangle$ averaged over the boundary layer, although the vertical profile comparisons
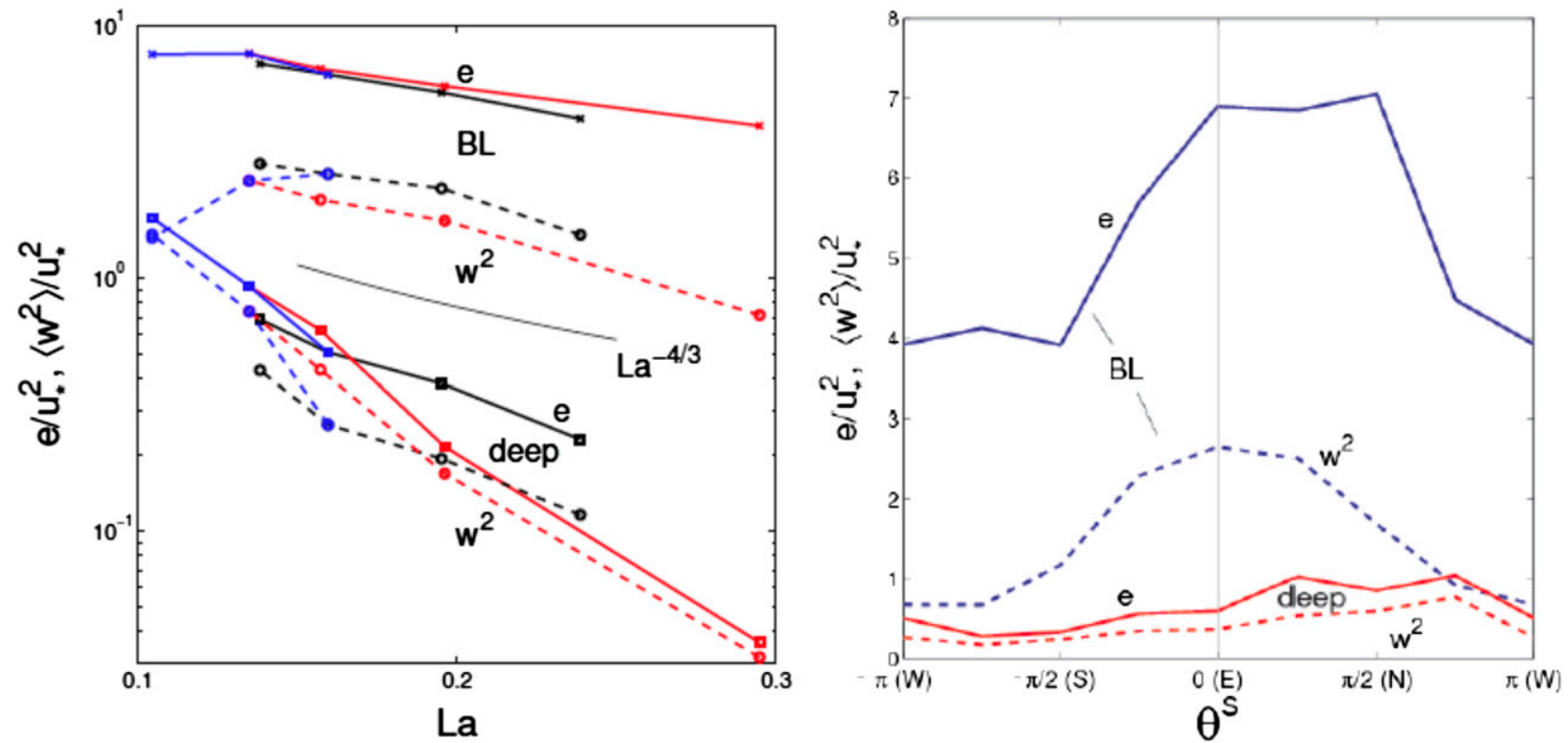

FIG. 8. Depth-averaged turbulent variances: $e$ (solid) and $\left\langle w^{2}\right\rangle$ (dashed) grouped separately for within the BL (the upper curves; $-h \leq$ $z \leq 0$ ) and for the deep layer below (the lower curves; $-H \leq z \leq-h$ ). (left) A function of La is for the case sets Us1 (black), Us2 (red), and Ua (blue). For comparison, a scaling curve $\sim \mathrm{La}^{-4 / 3}$ is also plotted (light black; see text). (right) A function of $\theta^{s}$ is for the case set $\Theta$ s with the BL averages in blue and the deep averages in red. 

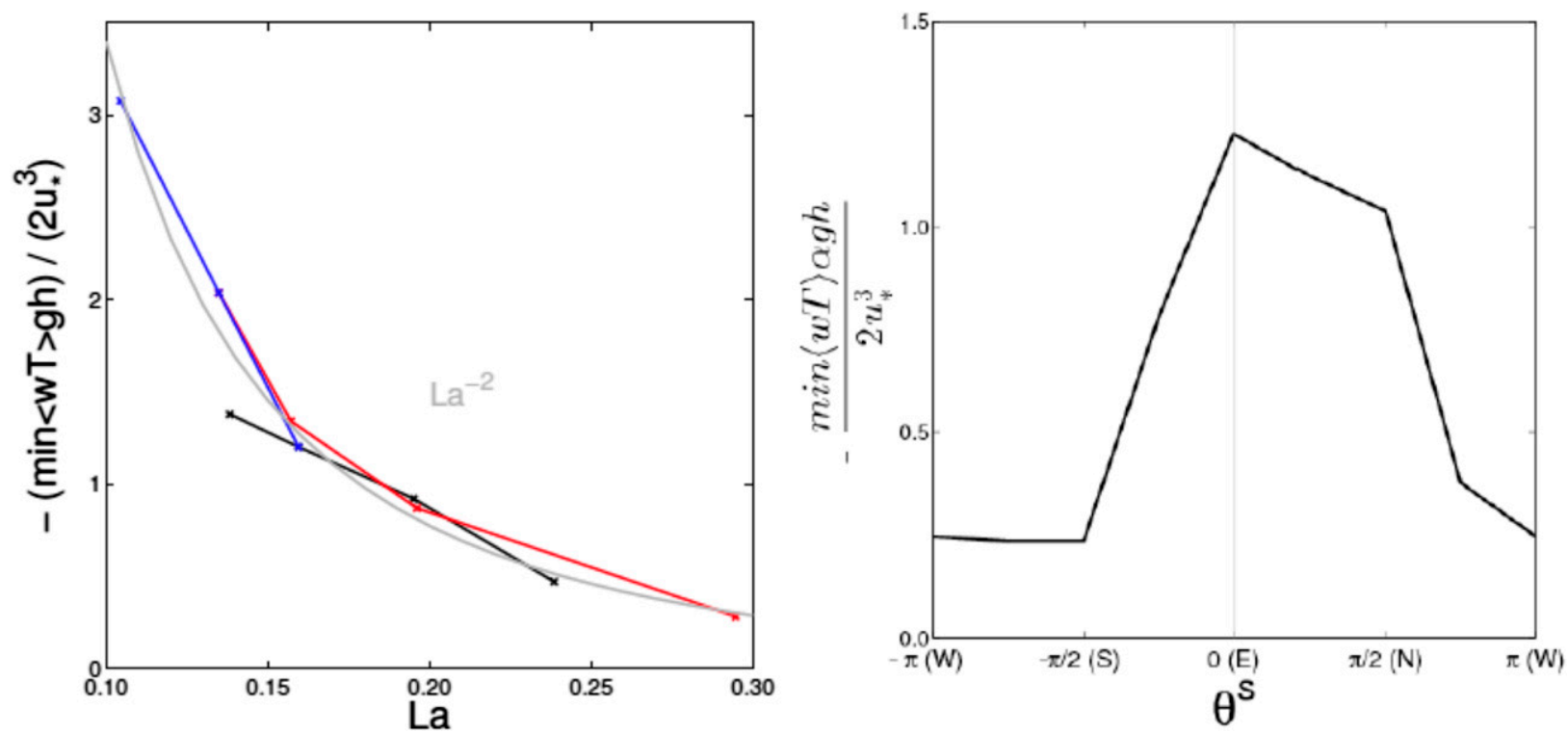

FIG. 9. Entrainment-layer temperature flux extremum in $z$, normalized by $2 u_{*}^{3} / \alpha g h$ (with thermal expansion coefficient $\alpha=2 \times$ $10^{-4}{ }^{\circ} \mathrm{C}^{-1}$ ), as a function of (left) La for case sets Us1 (black), Us2 (red), and Ua (blue) and as a function of (right) $\theta^{s}$ for case set $\Theta$ s. A comparison curve $\sim \mathrm{La}^{-2}$ (light gray) is drawn through the data end points (left).

for $\theta^{s}= \pm \pi / 2$ in Fig. 7 show that this symmetry is not precise. $^{5}$ The boundary layer $e$ measures in Figs. 7 and 8 are even farther away from $\theta^{s}$ symmetry than $\left\langle w^{2}\right\rangle$, with larger $e$ for $\theta^{s}>0$. Below the boundary layer, both $e$ and $\left\langle w^{2}\right\rangle$ are so asymmetric in $\theta^{s}$ that their maxima occur for northward swell (similar to the inertial current in Fig. 5). The $D^{s}$ dependencies in case set Ds (not shown) are rather slight, with some evident profile sensitivities in $e(z)$ and $\left\langle w^{2}\right\rangle(z)$ and a weak tendency for $e$ averaged over the boundary layer to increase as $D^{s}$ decreases.

Langmuir turbulence is known to increase the entrainment rate in a stratification-limited boundary layer (McWilliams et al. 1997) as a consequence of Langmuir circulations penetrating into the stable stratification and scooping colder water into the boundary layer. This is the process that causes $h(t)$ to increase in the present solutions.

The entrainment rate is associated with the negative minimum in the vertical temperature flux profile of $\langle w T\rangle(z)$. This temperature flux extremum is rescaled by its contribution to the change in potential energy PE associated with mixed layer deepening into a uniformly stratified interior and cooling of its average temperature $\bar{T}(t)$; that is,

\footnotetext{
${ }^{5}$ Van Roekel et al. (2012, their Fig. 8) shows a monotonic decrease in $\left\langle w^{2}\right\rangle(z)$ as a function of the Langmuir cell orientation angle, which is $\approx \theta^{s} / 2$ for their misaligned cases. See section 7 for further comparative remarks.
}

$$
\partial_{t} \mathrm{PE} \equiv-\frac{\alpha g h^{2}}{2} \partial_{t} \bar{T}=-\frac{\alpha g h}{2} \min _{z}[\langle w T\rangle(z)]
$$

and then normalized by the scaling estimate for the rate of wind work acting to increase the kinetic energy $u_{*}^{3}$; that is, $\langle w T\rangle$ is normalized by a combined factor of $2 u_{*}^{3} / \alpha g h$, with $\alpha$ as the thermal expansion coefficient. Figure 9 shows that added swell further enhances the entrainment rate, which increases as La decreases with a dependency that can be fit approximately as a linear function of $\mathrm{La}^{-2}$. The entrainment rate is largest when the swell is aligned with the wind-sea, with a similar $\theta^{s}$ dependency as found for turbulent intensity $e$ (i.e., larger for $\theta^{s}>0$ than for $\theta^{s}<0$; Fig. 8). Consistent with the $e$ change with $D^{s}$ remarked on above, there is a weak tendency for the minimum in $\langle w T\rangle$ to decrease in magnitude as $D^{s}$ decreases. Both $e$ and $\left\langle w^{2}\right\rangle$ are larger with Stokes drift than without it (case NB defined in Table 1; not shown). Thus, stronger Langmuir turbulence has stronger entrainment.

As expected, there is a strong correlation between entrainment rate and the increased value of $h$ averaged over the analysis period. Because of the strong thermocline, the changes in $h$ are modest, ranging from only a slight increase over the initial $h(0)=33 \mathrm{~m}$ in case NB up to a value of $36 \mathrm{~m}$ for the wind-aligned case in case set Us1 with the strongest wind and swell. Yet again, the $D^{s}$ dependencies for $\langle w T\rangle$ and $h(t)$ are modest, although there are small increases with $D^{s}$. 

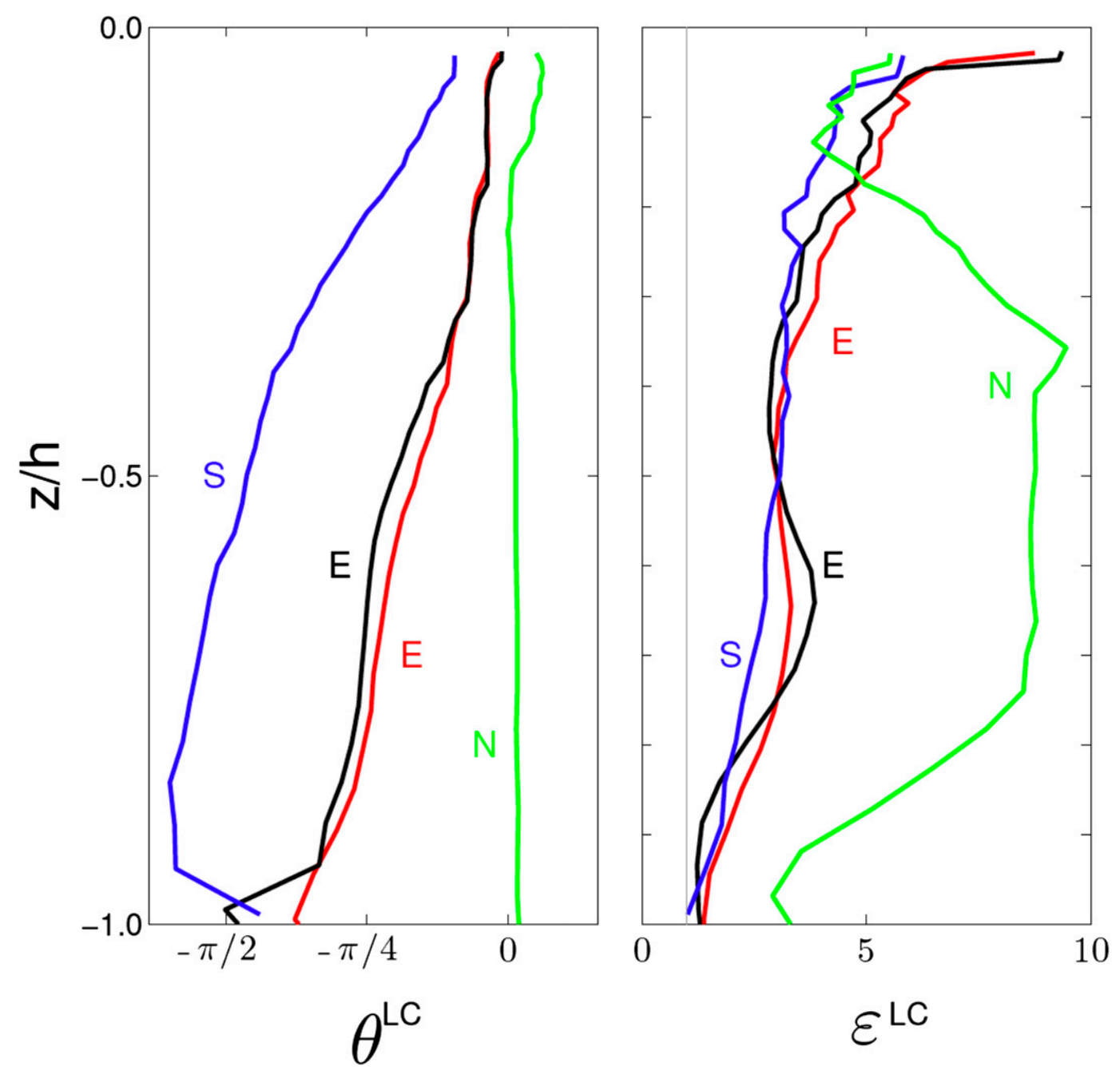

FIG. 10. Vertical profiles of the orientation angle of Langmuir circulations (left) $\theta^{\mathrm{LC}}(z)$ and their ellipticity (right) $\varepsilon^{\mathrm{LC}}(z)$ (ratio of major to minor axis) determined from fitting an ellipse to the horizontal correlation function $C_{w}$ for $w(x, y)$ at a fixed $z$ in (27). These are for the cases from the case sets Us1 with $U^{s}=0$ (black) and $\Theta$ s with $U^{s}=$ $0.25 \mathrm{~m} \mathrm{~s}^{-1}$ and $\theta^{s}$ oriented toward east (red), north (green), and south (blue).

How can the $\theta^{s}$ asymmetry in Figs. $8-10$ be explained? The $\mathbf{u}_{\text {st }}$ profiles are symmetric with respect to the sign of $\theta^{s}$, as part of the symmetry of the $\langle\mathbf{u}\rangle$ profiles in the Ekman-Stokes model (section 3). The answer must lie in an asymmetry in the turbulent dynamics (cf. the constant $\kappa_{o}$ assumption in Ekman-Stokes). A plausible cause is the difference in Eulerian-mean velocity $\langle\mathbf{u}\rangle(z)$ between positive and negative $\theta^{s}$. They are composed of an Ekman spiral in the southern quadrants to the right of the wind plus an anti-Stokes component opposite to $\mathbf{u}_{\mathrm{st}}(z)$. For northward swell $\left(\theta^{s}>0\right)$, the southward anti-Stokes component broadly reinforces the Ekman flow, whereas for southward swell, the anti-Stokes component is opposing. Therefore, $\langle\mathbf{u}\rangle$ is stronger for northward swell waves. We conjecture that this implies a greater growth rate for its linear shear instability that generates the turbulence. ${ }^{6}$ Support for this conjecture is provided in Table 2, which has the normalized and depth-integrated values for the rates of mean shear and Stokes turbulent kinetic energy production [(7) in McWilliams et al. (2012)]:

\footnotetext{
${ }^{6}$ To be specific we have in mind a calculation for given $1 \mathrm{D}$ profiles of $\langle\mathbf{u}\rangle(z), \mathbf{u}_{\mathrm{st}} z$, and $\langle T\rangle(z)$, with or without background eddy viscosity and diffusivity profiles. These would be specified from the mean state of LES solutions of the type presented here. In the case of nonrotating, unstratified flow, Craik and Leibovich (1976) and subsequent papers show that the Stokes vortex force enables a new shear instability mode as a paradigm for the emergence of Langmuir circulations. Such a calculation has not yet been performed for the conditions relevant here.
} 
TABLE 2. Turbulent kinetic energy production rates of (25), depth-integrated and normalized by $u_{*}^{3}$. They are grouped by $\theta^{s}$ magnitude for the case set $\Theta$ s.

\begin{tabular}{llrc}
\hline \multicolumn{1}{c}{ Case } & $\mathcal{P}_{u}$ & $\mathcal{P}_{\text {st }}$ & $\mathcal{P}_{u}+\mathcal{P}_{\text {st }}$ \\
\hline Us1: $U^{s}=0($ no swell) & 1.5 & 13.2 & 14.7 \\
$\Theta \mathrm{s}: \theta^{s}=0(\mathrm{E})$ & 3.2 & 21.3 & 24.5 \\
$\Theta \mathrm{s}: \theta^{s}=\pi / 4(\mathrm{NE})$ & 5.6 & 16.7 & 22.3 \\
$\Theta \mathrm{s}: \theta^{s}=-\pi / 4(\mathrm{SE})$ & 1.3 & 19.5 & 20.8 \\
$\Theta \mathrm{s}: \theta^{s}=\pi / 2(\mathrm{~N})$ & 6.1 & 9.5 & 15.6 \\
$\Theta \mathrm{s}: \theta^{s}=-\pi / 2(\mathrm{~S})$ & 0.8 & 12.9 & 13.7 \\
$\Theta \mathrm{s}: \theta^{s}=3 \pi / 4(\mathrm{NW})$ & 8.5 & 3.3 & 11.8 \\
$\Theta \mathrm{s}: \theta^{s}=-3 \pi / 4(\mathrm{SW})$ & 2.7 & 7.5 & 10.2 \\
$\Theta \mathrm{s}: \theta^{s}= \pm \pi(\mathrm{W})$ & 7.7 & 3.1 & 10.8 \\
\hline
\end{tabular}

$$
\begin{aligned}
& \mathcal{P}_{u}(z)=-\left\langle\mathbf{u}^{\prime} w^{\prime}\right\rangle \cdot \partial_{z}\langle\mathbf{u}\rangle \\
& \mathcal{P}_{\mathrm{st}}(z)=-\left\langle\mathbf{u}^{\prime} w^{\prime}\right\rangle \cdot \partial_{z} \mathbf{u}_{\mathrm{st}} .
\end{aligned}
$$

The mean shear production rate $\mathcal{P}_{u}$, in particular, is much larger for northward swell for a given value of $\left|\theta^{s}\right|$, so that the total production rate is also larger. In almost all cases, $\mathcal{P}_{u}<\mathcal{P}_{\text {st }}$, with westward swell as the only exception (where total production is small). Thus, stronger $\langle\mathbf{u}\rangle$ is associated with a stronger turbulent production rate, stronger turbulent intensity, and stronger entrainment, and even with a fatter Reynolds stress profile (Fig. 4), albeit only to a modest degree.

The total kinetic energy production (25) can alternatively be written using (17) and (19)-(21) as

$$
\begin{aligned}
\mathcal{P} & \equiv \mathcal{P}_{u}+\mathcal{P}_{\text {st }}=-\left\langle\mathbf{u}^{\prime} w^{\prime}\right\rangle \cdot \partial_{z}\left\langle\mathbf{u}^{L}\right\rangle \\
& =\kappa^{L}\left|\partial_{z} \mathbf{u}^{L}\right| \cos \left[\theta^{L}\right]=\frac{1}{f}\left|\left\langle\mathbf{u}^{\prime} w^{\prime}\right\rangle\right|\left|\partial_{z}^{2}\left\langle\mathbf{u}^{\prime} w^{\prime}\right\rangle\right| \cos \left[\theta^{L}\right] .
\end{aligned}
$$

Thus, the energy production is positive if $\cos \left[\theta^{L}\right]>0$, as it is at all depths in Fig. 6, and it is proportional to the product of the Reynolds stress and its profile curvature. As discussed at the end of section 4, both of these factors are increasing as La decreases in the swell regime (i.e., $\mathrm{La} \leq 0.3$ ). This is consistent with energy production and velocity variance strongly increasing (Fig. 8), even while their ratio $\kappa L$ in (21) is decreasing (Fig. 6). Even for weak waves with $\mathrm{La}>1$, the decrease in $\left|\partial_{z} \mathbf{u}^{L}\right|$ is more than countered by the increase in $\left|\left\langle\mathbf{u}^{\prime} w^{\prime}\right\rangle\right|$ (i.e., $\kappa^{L}$ increases), so that $\mathcal{P}$ also increases with decreasing La in this regime. The increasing $\mathcal{P}$ is the plausible cause for the increasing $e$ and entrainment as La decreases across its entire range.

Finally, it can be remarked that Langmuir turbulence differs from shear turbulence in that the negative feedback between the turbulent kinetic energy production and the mean shear is not as tight: $\mathcal{P}_{u}$ is proportional to $\partial_{z}\langle\mathbf{u}\rangle$, which in the absence of waves will be reduced if $e$ and momentum mixing increase, whereas $\mathcal{P}_{\text {st }}$ is proportional to $\partial_{z} \mathbf{u}_{\mathrm{st}}$, which is not as directly reduced by changes in the boundary layer turbulence, but would be so only if the feedback on the wave field, here neglected, were strong enough. This is a partial explanation for the strong inverse dependence of $e$ on La in Langmuir turbulence.

\section{Langmuir circulations}

Langmuir circulations in a nonrotating, unstratified surface layer have an idealized shape of closely packed, longitudinal roll cells, orienting with the surface stress and wind-sea and extending vertically throughout the boundary layer (Leibovich 1983). In equilibrium Langmuir turbulence (McWilliams et al. 1997), they have more fragmented shapes but still retain appreciable horizontal anisotropy; they are rotated to the right of the wind-sea increasingly with depth. At sea they are most easily visualized by buoyant debris trapped in surface convergence lines, and in LES solutions the horizontal pattern of $w$ (especially $w<0$ ) is a comparably useful visualization.

Statistical measures of the intensity and entrainment efficacy of Langmuir circulations show strong dependencies on swell-wave amplitude and orientation (section 6). How does their spatial structure change with swell? To analyze the horizontal structure of the Langmuir circulations, a spatial correlation function ${ }^{7}$ is calculated from $w(x, y)$ at each height $z$ :

$C_{w}(\xi, \eta, z)=\frac{\langle w(x+\xi, y+\eta, z, t) w(x, y, z, t)\rangle}{\left\langle w(x, y, z, t)^{2}\right\rangle}$,

where again the angle brackets denote an average over all $(x, y$, and $t)$. Langmuir circulations have horizontally elongated patterns, which are expressed in $C_{w}$ as approximately elliptical contours decreasing away from the central extremum where $C_{w}(0,0, z)=1$. Therefore, an ellipse is fit to an intermediate contour of $C_{w}$ in the $(\xi, \eta)$ plane (e.g., $C_{w}=0.4$ for Fig. 10$) .{ }^{8}$ The direction of its major axis is designated as $\theta^{\mathrm{LC}}(z)$ and the ratio of its major and minor axes as $\varepsilon^{\mathrm{LC}}(z)$. Respectively, these quantities measure the mean orientation angle and degree of elongation (anisotropy) of the Langmuir circulations. The resulting profiles of these measures are shown in Fig. 10 for several cases in case sets Us1 and $\Theta$ s. For

\footnotetext{
${ }^{7}$ An alternative statistical measure of Langmuir circulation orientation is the direction determined by a peak in the distribution function for $\tan ^{-1}\left(\omega^{y} / \omega^{x}\right)$, where $\omega$ is the vorticity vector (see, e.g. Van Roekel et al. 2012). This gives similar results to the correlation function method used here, but with somewhat greater estimation noise.

${ }^{8}$ The elliptical fit parameters are only weakly dependent on the $C_{w}$ value as long as $\varepsilon^{\mathrm{LC}}$ is not too close to 1 .
} 
swell aligned with the wind-sea, $\theta^{\mathrm{LC}}(z)$ is nearly wind aligned at the surface and progressively rotates clockwise with depth, while $\varepsilon^{\mathrm{LC}}(z)$ is largest at the surface and decreases with depth down to near isotropy at the bottom of the boundary layer. Neither of these behaviors is very different due to the presence of swell. However, when the swell is misaligned with the wind-sea, the Langmuir circulations rotate in the direction of the swell by a small amount near the surface. Below the surface, the behavior of $\theta^{\mathrm{LC}}(z)$ is very different for $\theta^{s}= \pm \pi / 2$ : it rotates clockwise even more rapidly for southward swell, and it is nearly independent of depth for northward swell. Additionally, for northward swell, the circulation anisotropy is very much enhanced in the middle of the layer, compared to the surface and compared to the other $\theta^{s}$ orientations.

Beyond these statistical measures, the Langmuir circulation patterns are displayed in Fig. 11 for visual assessment. Near the surface $(z=-1 \mathrm{~m})$ the patterns are only moderately different for different values of $U^{s}$ and $\theta^{s}$, although some variations in $w$ amplitude and anisotropy direction are discernible. By the middle of the layer $(z=-10 \mathrm{~m})$, however, the pattern differences are quite large. Besides simple amplitude dependencies for $w$-larger $w$ both for larger $U^{s}$ and for $\theta^{s}$ in the range from SE to $\mathrm{N}$ - the nonmonotonic dependency of $\theta^{\mathrm{LC}}$ on $\theta^{s}$ and the highly variable degrees of pattern complexity are notable. For $U^{s}=0$, the pattern is a familiar one from previous studies of equilibrium Langmuir turbulence. For an opposing swell $\left(\theta^{s}=\pi\right)$, the Langmuir circulations are regular roll cells with nearly uniform spacing and a north-south orientation perpendicular to both the wind-sea and the swell-sea. But with the swell in the SE-N sector, there are multiple Langmuir circulation orientations and scales and complicated branching patterns from a dominant $w$ extremum. It is clear that no simple conception of the structure of Langmuir circulations holds across the range of swell conditions.

In Van Roekel et al. (2012), considerable attention is given to the near-surface orientation angle of Langmuir circulations $\theta^{\mathrm{LC}}$ as the misalignment angle between an equilibrium wind-sea and the wind stress is varied over a range from $\theta^{m}=0$ to $+3 \pi / 4$. The principal motivation is to interpret the dependency of $\left\langle w^{2}\right\rangle$ on the wave parameters (further discussed in section 8 ). Their result is that $\theta^{\mathrm{LC}}$ monotonically increases with $\theta^{m}$ (their Figs. 2 and 7). In that paper several statistical predictors of $\theta^{\mathrm{LC}}$ are assessed, with varying degrees of skill, and mostly $\theta^{\mathrm{LC}}$ is about half way between the wind and wave angles for this set of cases. In Fig. 10, the sign of $\theta^{\mathrm{LC}}$ near the surface is the same as the sign of $\theta^{s}$, but its magnitude is much less than $\theta^{s} / 2$. In the boundary layer interior, the $\theta^{\mathrm{LC}}(z)$ profile shapes are quite different in the north and south cases. Furthermore, the Langmuir circulation pattern complexity in Fig. 11 is not always well characterized by only a single angle, even at a fixed depth. Therefore, $\theta^{\mathrm{LC}}$ does not seem to be a robust property of Langmuir turbulence with different swellwave orientations.

The Langmuir circulation pattern dependencies are not strong with $D^{s}$ variations in case set Ds (again not shown), except for a moderate enhancement of the $w$ magnitudes near the surface in the case with the smallest $D^{s}$ value of $4 \mathrm{~m}$ (i.e., a steep swell wave due to its short wavelength of $\lambda=50 \mathrm{~m}$ ).

A further structural oddity emerges in the limit of large swell and weak wind (i.e., in the case set Ua for the smallest $U_{a}$ value of $2.5 \mathrm{~m} \mathrm{~s}^{-1}$; Fig. 12). The Langmuir circulations become much larger in scale, both in their along-axis extent and in the spacing between neighboring circulations. In this case, they are bundles of anisotropic overturning cells rather than individual isolated cells. Within the bundles are multiple spatial scales and orientation angles. A primary orientation-roughly northwest (NW)-SE at both depths shown in the figurecharacterizes the long axis of the bundle, but different orientations arise on a finer scale within the bundlesmore E-W near the surface and more N-S in the boundary layer interior. This is certainly a highly organized turbulent coherent structure, but it is a long way from an idealized roll cell. For the smallest La values in Fig. 9, some sort of regime transition is occurring, with a break from the $\sim \mathrm{La}^{-4 / 3}$ scaling growth of $\left\langle w^{2}\right\rangle$ in the boundary layer, while the scaling growth of $\left\langle w^{2}\right\rangle$ below the boundary layer continues, as does the growth of $\langle w T\rangle$ (Fig. 9). Any changes in the growth of $e$ are less clear.

Comparison of the statistics and patterns among the cases in set Lh indicate that there is only a weak dependency on the domain width $L$, with the widest domain $(L=1200 \mathrm{~m})$ large enough to encompass several of the large-scale Langmuir circulations, suggesting a convergence of the LES solutions with this essentially computational parameter. If solutions were sought with even smaller La values (e.g., even weaker winds), it is likely that the Langmuir circulation size would grow even larger, requiring larger $L$. Malecha et al. (2013) have proposed a multiscale asymptotic treatment in the limit of $\mathrm{La} \rightarrow 0$ as an aid to computational efficiency; it is an open question whether this is a viable approach to dealing with the complex bundle structures evident in Fig. 12.

\section{Summary and discussion}

The presence of remotely generated swell waves significantly alters the Langmuir turbulence of an equilibrium wind-sea. The Lagrangian-mean flow profile $\mathbf{u}^{L}(z)$ 

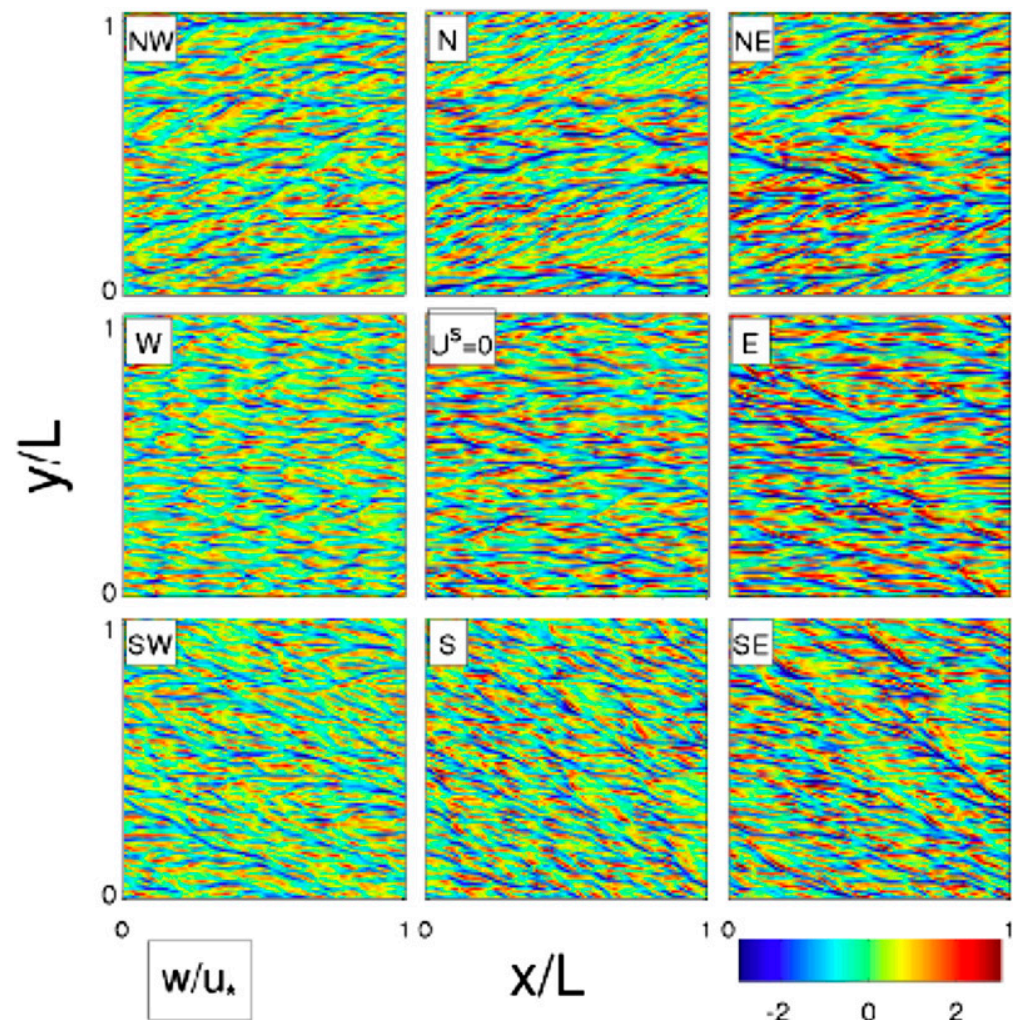

10
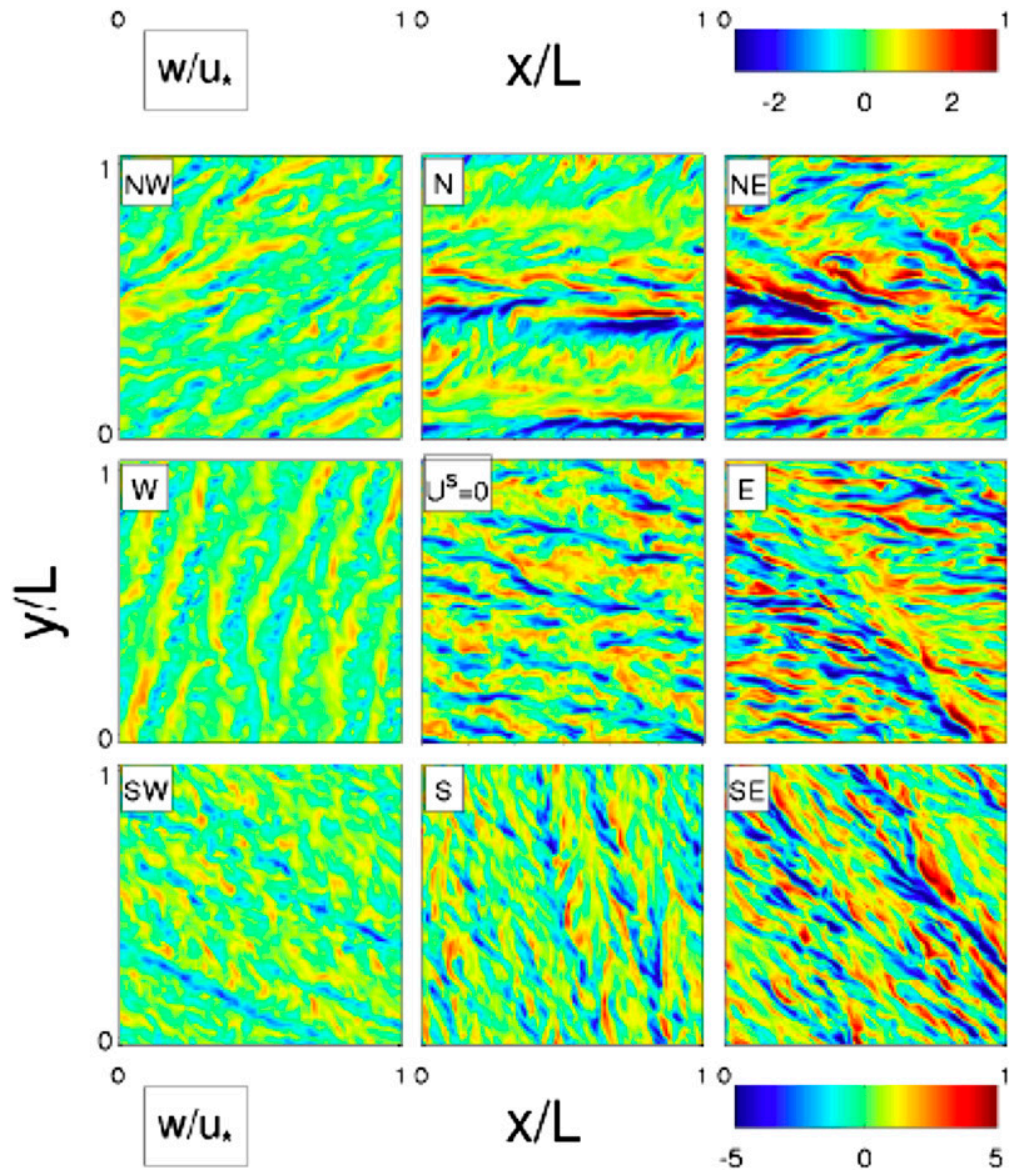

FIG. 11. The $w(x, y)$ snapshots at $z=$ (top) -1 and (bottom) $-10 \mathrm{~m}$, normalized by $u_{*}$. In the center is the case from the set Us1 with no swell $\left(U^{s}=0\right)$, and around the edge are the cases in set $\Theta \mathrm{s}\left(U^{s}=0.25 \mathrm{~m} \mathrm{~s}^{-1}, D^{s}=16 \mathrm{~m}\right)$ located in their appropriate compass positions. 

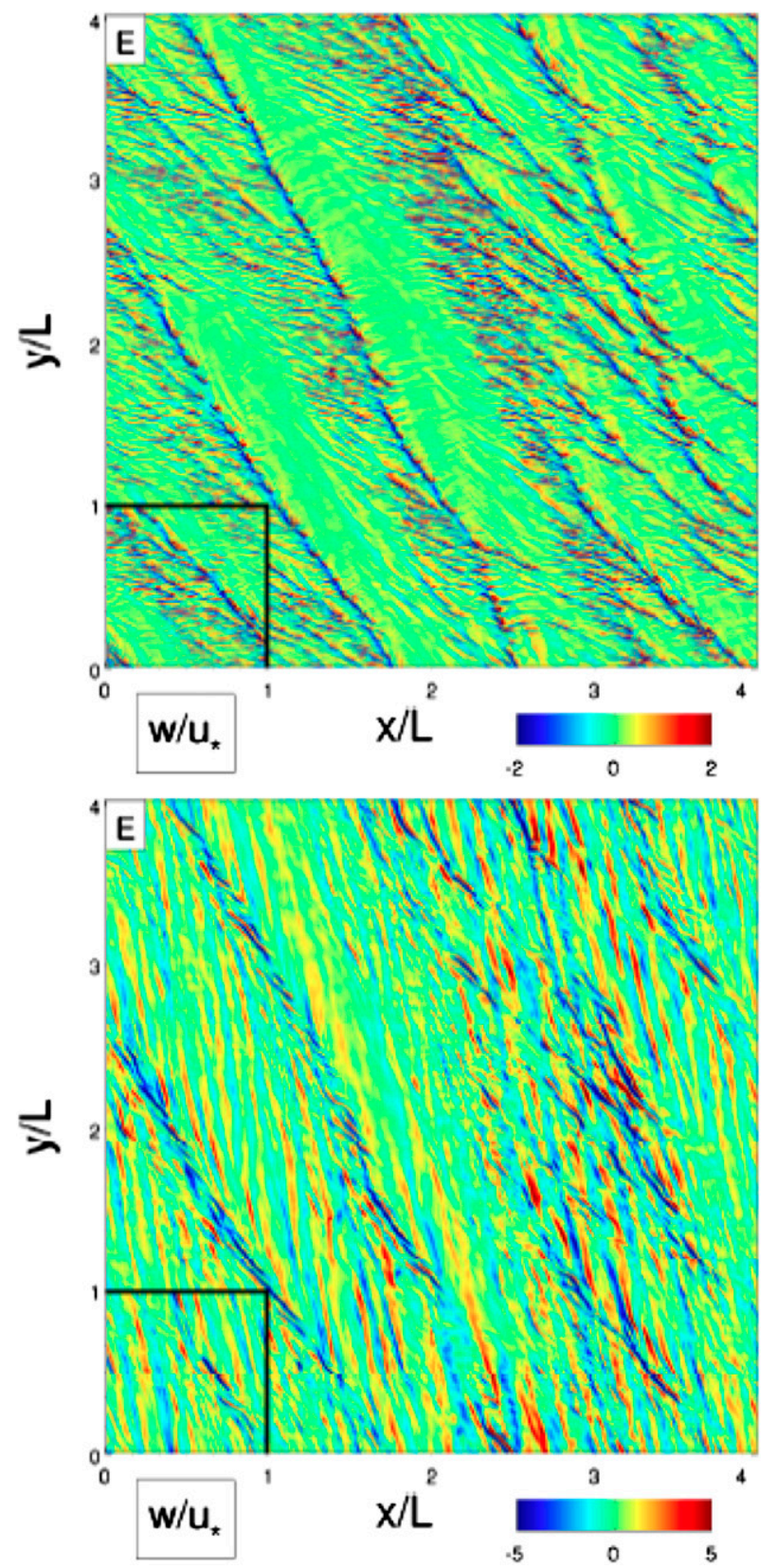

FIG. 12. The $w(x, y)$ snapshots at $z=$ (top) -1 and (bottom) $-10 \mathrm{~m}$ for the case set Lh (i.e., $U_{a}=2.5 \mathrm{~m} \mathrm{~s}^{-1}, U^{s}=0.25 \mathrm{~m} \mathrm{~s}^{-1}$, $\theta^{s}=0, D^{s}=16 \mathrm{~m}$, and variable $L$ ) with $\mathrm{La}=0.11$. The full panel is for the quadrupled horizontal domain case with $L=1200 \mathrm{~m}$, and the lower-left inset is for the standard domain with $L=300 \mathrm{~m}$ at an uncorrelated time.

has a larger surface value and a fatter Ekman spiral throughout the boundary layer with increasing swell magnitude $U^{s}$. With misaligned wind and swell, $\mathbf{u}^{L}$ rotates toward the swell direction, but only within a limited angular range that is almost equal to $\pm 30^{\circ}$. The Eulerianmean flow $\langle\mathbf{u}\rangle(z)$ exhibits an anti-Stokes component that becomes large when $U^{s}$ is large, but this cancellation is not so complete as to leave $\mathbf{u}^{L}$ unaltered with added swell-waves. The Reynolds stress profile $\mathbf{R}(z)$ also has a fatter Ekman spiral with strong swell, and with misalignment it rotates to a moderate degree toward the swell direction within the boundary layer interior. For a given swell magnitude, the orientation of opposing wind- and swell-seas (i.e., $\theta^{s}= \pm \pi$ ) yields the weakest $\mathbf{u}^{L}$ and thinnest Ekman spiral. All of these behaviors are similar to the analytic Ekman-Stokes model solutions with constant density and eddy viscosity (section 3 ).

Turbulent intensity, $e$ and $\left\langle w^{2}\right\rangle$, and entrainment rate $\langle w T\rangle$ are inverse functions of Langmuir number La. Within the boundary layer the intensity measures scale roughly as $\mathrm{La}^{-4 / 3}$, consistent with an argument based on Stokes production as the dominant source of Langmuir turbulence, while for the inertial current, entrainment rate, and fluctuation variances below the boundary layer, the dependencies are even steeper, perhaps $\sim \mathrm{La}^{-2}$ (as predicted for $U_{\text {in } 0}$ by the Ekman-Stokes model).

The Ekman-Stokes model predicts a reflection symmetry in the swell direction (i.e., independence of the sign of $\theta^{s}$ ) for the mean boundary layer, but this does not hold in the LES solutions. While $\mathbf{u}^{L}(z)$ is approximately symmetric in its weakening and rotation for positive and negative $\theta^{s}$ (Fig. 3, right), most other boundary layer properties are asymmetric. This is strongly so for inertial current amplitude, turbulent intensity, and entrainment rate, all of which are much stronger with leftward $\left(\theta^{s}>0\right.$; counterclockwise, anticyclonic, and northward) swell misalignment than with rightward misalignment. The Langmuir circulation patterns are similarly asymmetric with $\theta^{s}$, with stronger, more vertically aligned, and more elongated eddies when $\theta^{s}>0 .{ }^{9}$ As La decreases with larger swell, the Langmuir circulation patterns become more complex and multiscale; at the smallest value of $\mathrm{La} \approx 0.1$ included here, the outermost longitudinal and transverse correlation scales become very large compared to the boundary layer depth (Fig. 12).

Even with a large added swell, however, the general characteristics of Langmuir turbulence are similar to what occurs in the equilibrium wind-sea regime, but the quantitative differences are not small. In the swell-wave parameter space of $\left(U^{s}, \theta^{s}, D^{s}\right)$, the most important parameter is La in (3). With aligned swell, as $U^{s}$ increases and La decreases, the Lagrangian-mean flow, turbulent intensity both within the boundary layer and below, and entrainment rate all increase. As $\theta^{s}$ varies away from wind alignment, La in (3) increases due to swell- and

\footnotetext{
${ }^{9}$ The nonequilibrium misalignment cases examined in Van Roekel et al. (2012) all have nonnegative wave rotation angles relative to the wind $\theta$ equal to or greater than zero.
} 
wind-sea Stokes drift misalignment. This increase in La accounts partly for the general tendencies for decreases in the preceding quantities; however, the $\theta^{s}$ dependency is asymmetric in its sign in the LES (unlike in the Ekman-Stokes model or in the La definition), and sometimes the asymmetry is strong enough to overcome the implied change due to La (e.g., the peak value for $e$ averaged over the boundary layer occurs at $\theta^{s}=\pi / 2$ in Fig. 8). The $D^{s}$ dependencies are mostly rather weak, although inertial amplitude $U_{\text {in } 0}$ and diagnosed eddy viscosity $\boldsymbol{\kappa}^{L}(z)$ are exceptions. This is consistent with the absence of any $D^{s}$ influence in the definition of La in (3), which differs from the alternative definition in Harcourt and D'Asaro (2008) in this aspect.

In Van Roekel et al. (2012, Fig. 12) functional fits are presented for $\left\langle w^{2}\right\rangle$ from LES cases as alternative functions of alternatively defined Langmuir numbers that combine the influences of Stokes drift magnitude, direction, and depth scale. The latter alternatives include different depth weighting for the Stokes drift magnitude in the denominator [as originally proposed by Harcourt and D'Asaro (2008) to exclude dependencies on the near-surface wind-sea $\mathbf{u}_{\mathrm{st}}$ magnitude and on its extension beyond the boundary layer when $D^{s}>$ $h$ ] and different angular projections for the misaligned swell, wind, and Langmuir circulations. While all of the fits are somewhat skillful, the skill levels among the alternatives are not sharply distinguished. The swell influences summarized in the preceding paragraph do not accurately collapse into a universal function of any of these alternative Langmuir numbers (not shown). In particular, the orientation angle $\theta^{\mathrm{LC}}$ is a complex function of $\theta^{s}$ and depth (Figs. 10 and 11); hence, it is not useful as an a priori projection angle for a composite $\mathrm{La}$.

Oceanic circulation models require a boundary layer parameterization because their computational grids are necessarily too coarse to resolve Langmuir turbulence. With data inputs about the surface fluxes and gravity wave field, the desired outcome is accurate profiles of $\langle\mathbf{u}\rangle(z)$ [or $\left.\mathbf{u}^{L}(z)\right],\langle T\rangle(z)$, and other scalar profiles determined in competition with larger-scale dynamical influences, while accurate characterizations of turbulent quantities such as $e,\left\langle w^{2}\right\rangle$, and $\kappa(z)$ are only means to that end. In particular, the swell influences demonstrated here do need to be parameterized.

For $\mathbf{u}^{L}(z)$ and the inertial current amplitude, even the simple Ekman-Stokes model with constant eddy viscosity is fairly skillful (Figs. 3, 4, and 5), even though in practice the parameterization must also account for the influence of stratification in limiting the boundary layer depth and the generally convex shape of the eddy viscosity profile [both of which are parts of the KPP scheme; Large et al. (1994)]. On the other hand, the complexity of the swell influences on the eddy viscosity profile diagnosed from the LES solutions [i.e., $\boldsymbol{\kappa}^{L}(z)$ in Fig. 6] indicates that neither the constant $\kappa_{O}$ in the Ekman-Stokes model nor the $[K(z), 0]$ in the KPP scheme would yield a highly accurate $\mathbf{u}^{L}(z)$. In particular, the magnitude of $\kappa^{L}$ increases in equilibrium wind-wave Langmuir turbulence compared to shear turbulence without waves (McWilliams and Sullivan 2000; McWilliams et al. 2012), but as La further decreases with swell waves, the eddy viscosity magnitude decreases (section 4); that is, $|\boldsymbol{\kappa}|$ must have a maximum at an intermediate value of $\mathrm{La}$ near its wind-sea equilibrium value of 0.3 . These results raise a subtle design issue about the trade-off between parameterization simplicity (which often correlates with robustness across regimes) and $\langle\mathbf{u}\rangle(z)$ profile accuracy from the perspective of practical utility in circulation models. This decrease in $|\boldsymbol{\kappa}|$ for very small La happens even while $e$ and $\left\langle w^{2}\right\rangle$ continue to increase with decreasing La because of increasing turbulent kinetic energy production (section 6); these opposing tendencies contradict the mixing length representation of $\kappa \sim w_{s} h$, with $w_{s}$ as a turbulent velocity scale. Our present view is that relatively simple rules for the dependency of $\kappa(z)$ on the wave parameters (i.e., simpler than manifested in Fig. 6) may provide a useful accuracy for $\langle\mathbf{u}\rangle(z)$.

For $\langle T\rangle(z)$ and other material properties, the important parameterization target of Langmuir turbulence is the entrainment rate (Fig. 9). Its dependencies on the swell parameters are fairly simple, and they mirror those of the depth-averaged turbulent intensity (Fig. 8). The entrainment rate, represented in the KPP scheme as the negative peak in $\langle w T\rangle(z)=-K_{s}(z) \partial_{z}\langle T\rangle(z)$ near the top of the thermocline, is a sensitive function of the eddy diffusivity profile near the bottom of the boundary layer, most importantly how far the diagnosed boundary layer depth at $z=-h$ penetrates into the stable stratification. The determination of $h$ is represented in the KPP scheme for the free convection regime by a critical bulk Richardson number condition where the mean velocity scale is augmented by a turbulent velocity scale $V_{t} \cdot{ }^{10}$ The

\footnotetext{
${ }^{10}$ In present implementations of the KPP scheme in circulation models, there are two alternative formulations for the critical Richardson number condition that determines the boundary layer depth $h$. One formulation is expressed as a ratio of the bulk differences across the boundary layer of buoyancy change and velocity change squared; in this representation $V_{t}^{2}$ is added to $\Delta\left\langle\mathbf{u}^{2}\right\rangle$ in the denominator (Large et al. 1994, section 3). The other formulation is expressed as a boundary layer integral condition of the competition between the destabilizing influence of $\left(\partial_{z}\langle\mathbf{u}\rangle\right)^{2}$ and the stabilizing influence of positive buoyancy stratification $\alpha g \partial_{z}\langle T\rangle$; in this representation, $V_{t}^{2} / h$ is added to the vertical integral of the mean shear variance (Lemarie et al. 2012, section 3). Both formulations have essentially the same dynamical rationale.
} 
rationale is that entrainment by encroachment into the thermocline can happen even when the mean shear is weak, as in convection at low wind speed or as in Langmuir turbulence with strong waves and weak winds. To choose $V_{t}$, one must specify the desired entrainment rate that will result from the KPP scheme when $V_{t}$ is large enough to dominate the mean shear in determining $h$. For free convection, the entrainment rule is the peak value of the pycnocline buoyancy flux, which is a negative fraction, $\beta_{T} \approx-0.15$, of the destabilizing surface upward buoyancy flux $\mathbf{B}>0$. For Langmuir turbulence with swell, the entrainment rule is expressed by the normalized functional dependencies plotted as the ordinates in Fig. 9, which are denoted as $F\left(U^{s}, \theta^{s}\right)$. With these rules a combined expression can be derived for $V_{t}$ with both convection and Langmuir turbulence, following the arguments in Large et al. (1994, p. 372); namely,

$$
V_{t}^{2}=\frac{C_{v} N h w_{s}}{\operatorname{Ri}_{\mathrm{cr}}}\left[\frac{\left(-\beta_{T}\right) w_{*}^{3}+2 u_{*}^{3} \mathcal{F}}{w_{s}^{3}}\right]^{1 / 2} .
$$

The notation is as in that paper: $N$ is the buoyancy frequency just below the boundary layer; $w_{s}$ is the turbulent velocity scale used in scaling the eddy diffusivity for material concentrations, $K_{s}(z) \sim w_{s} h ; \mathrm{Ri}_{\text {cr }}$ is the critical value for the bulk Richardson number; $w_{*} \sim(B h)^{1 / 3}$ is the conventional convective velocity scale determined from the surface buoyancy flux and boundary layer depth; and $C_{v}$ is an empirical coefficient slightly larger than the one related to the shape of $\langle T\rangle(z)$ in the entrainment layer. The first term in the second numerator is the previously proposed convective contribution, and the second term is the new Langmuir contribution. Interpreting the result of Fig. 9 (left) as an experimental scaling relation for the normalized entrainment rate of $F \sim \mathrm{La}^{-2}$, the scaling dependency of $V_{t}$ from (28) in Langmuir turbulence is

$$
V_{t} \sim\left(N h u_{*}\right)^{1 / 2} \mathrm{La}^{-1 / 3},
$$

assuming that $w_{s} \sim u_{*} \mathrm{La}^{-2 / 3}$ (i.e., the scaling curve in Fig. 8).

The parameterization discussion in the preceding two paragraphs sketches how the KPP scheme could be modified to include the important effects of both a windsea and swell in Langmuir turbulence by generalizing $V_{t}$ in the condition determining $h$ and by choosing the turbulent velocity scale $w_{s}$ to have an appropriate dependency on the wave parameters La and $\theta^{s}$ (and perhaps $D^{s}$ ). However, a complete and implementable parameterization scheme requires extensive testing, and that task is beyond the scope of this paper.
In conclusion, the two relevant idealized regimes of equilibrium Langmuir turbulence in the ocean-with both wind and waves held steady in time and the turbulence evolving into a stationary state-are for a windsea alone, as previously analyzed in many papers, and for the addition of a remotely generated swell-sea, as analyzed here. Beyond these, the relevant regimes are inherently transient in both the winds and waves, and often the turbulence as well. Only a few transient situations have been addressed, for example, spinup from rest, the passage of a hurricane utilizing a transient, nonlocal wave model (Sullivan et al. 2012), and combined measurements of variable waves and currents (Smith 1992, 1998). Therefore, to make further progress it is of pressing importance to measure and model across a wide range of transient situations to discover how they differ from equilibrium Langmuir turbulence.

Acknowledgments. This research was sponsored by the National Science Foundation (Grant DMS-7850723757) and Office of Naval Research (Grant N00014-08-1-0597). Computations were performed on the supercomputers Bluefire at NCAR and Thresher, Trestles, and Gordon at SDSC. We appreciate Dr. Leonel Romero's guidance on the theory and observations of swell-waves and his help in preparing Fig. 1.

\section{REFERENCES}

Alves, J. H. G. M., 2006: Numerical modeling of ocean swell contributions to the global wind-wave climate. Ocean Modell., 11, 998-112.

, M. L. Banner, and I. R. Young, 2003: Revisiting the PiersonMoskowitz asymptotic limits for fully developed wind waves. J. Phys. Oceanogr., 33, 1301-1323.

Belcher, S. E., 2012: A global perspective on Langmuir turbulence in the ocean surface boundary layer. Geophys. Res. Lett., 39, L18605, doi:10.1029/2012GL052932.

Craik, A. D. D., and S. Leibovich, 1976: Rational model for Langmuir circulations. J. Fluid Mech., 73, 401-426.

Depley, M. T., F. Ardhuin, F. Collard, and B. Chapron, 2010: Space-time structure of long ocean swell fields. J. Geophys. Res., 115, C12037, doi:10.1029/2009JC005885.

Grant, A. L. M., and S. E. Belcher, 2009: Characteristics of Langmuir turbulence in the ocean mixed layer. J. Phys. Oceanogr., 39, 1871-1887.

Hanley, K. E., S. E. Belcher, and P. P. Sullivan, 2010: A global climatology of wind-wave interaction. J. Phys. Oceanogr., 40, 1263-1282.

Harcourt, R. R., 2013: A second-moment closure model of Langmuir turbulence. J. Phys. Oceanogr., 43, 673-697.

, and E. A. D'Asaro, 2008: Simulation of Langmuir turbulence in pure wind seas. J. Phys. Oceanogr., 38, 1542-1562.

Large, W. G., J. C. McWilliams, and S. Doney, 1994: Oceanic vertical mixing: A review and a model with a nonlocal boundary layer parameterization. Rev. Geophys., 32, 363-403.

Leibovich, S., 1983: The form and dynamics of Langmuir circulations. Annu. Rev. Fluid Mech., 15, 391-427. 
Lemarie, F., J. Kurian, A. F. Shchepetkin, M. J. Molemaker, F. Colas, and J. C. McWilliams, 2013: Are there inescapable issues prohibiting the use of terrain-following coordinates in climate models? Ocean Modell., 42, 57-79.

Malecha, Z., G. Chini, and K. Julien, 2013: A multiscale algorithm for simulating spatially-extended Langmuir circulation dynamics. J. Comput. Phys., in press.

Masson, D., 1993: On the nonlinear coupling between swell and wind waves. J. Phys. Oceanogr., 23, 1249-1258.

McWilliams, J. C., and P. P. Sullivan, 2000: Vertical mixing by Langmuir circulations. Spill Sci. Technol. Bull., 6, 225-237.

,-- , and C. H. Moeng, 1997: Langmuir turbulence in the ocean. J. Fluid Mech., 334, 1-30.

— E. Huckle, J. Liang, and P. Sullivan, 2012: The wavy Ekman layer: Langmuir circulations, breakers, and Reynolds stress. J. Phys. Oceanogr., 42, 1793-1816.

Polton, J. A., J. A. Smith, J. A. MacKinnon, and A. E. TejadaMartinez, 2008: Rapid generation of high-frequency internal waves beneath a wind and wave forced oceanic surface mixed layer. Geophys. Res. Lett., 35, L13602, doi:10.1029/ 2008GL033856.

Price, J. F., 1981: Upper ocean response to a hurricane. J. Phys. Oceanogr., 11, 153-175.

Romero, L., and W. K. Melville, 2010: Airborne observations of fetch-limited waves in the Gulf of Tehuantepec. J. Phys. Oceanogr., 40, 441-456.
Smith, J. A., 1992: Observed growth of Langmuir circulation. J. Geophys. Res., 97 (C4), 5651-5664.

1998: Evolution of Langmuir circulation during a storm. J. Geophys. Res., 103 (C6), 12668-12694.

Sullivan, P. P., and J. C. McWilliams, 2010: Dynamics of winds and currents coupled to surface waves. Annu. Rev. Fluid Mech., $\mathbf{4 2}$, $19-42$.

, _ _ , and W. K. Melville, 2007: Surface gravity wave effects in the oceanic boundary layer: Large eddy simulation with vortex force and stochastic breakers. J. Fluid Mech., 593, 405-452.

— J. B. Edson, T. Hristov, and J. C. McWilliams, 2008: Largeeddy simulations and observations of atmospheric marine boundary layers above nonequilibrium surface waves. $J$. Atmos. Sci., 65, 1225-1245.

—, L. Romero, J. C. McWilliams, and W. K. Melville, 2012: Transient evolution of Langmuir turbulence in ocean boundary layers driven by hurricane winds and waves. J. Phys Oceanogr., 42, 1959-1980.

Van Roekel, L. P., B. Fox-Kemper, P. P. Sullivan, P. E. Hamlington, and S. R. Haney, 2012: The form and orientation of Langmuir cells for misaligned wind and waves. J. Geophys. Res., 117, C05001, doi:10.1029/2011JC007516.

Young, I. R., 1999: Wind Generated Ocean Waves. Elsevier, 287 pp.

Zakharov, V. E., 2005: Theoretical interpretation of fetch-limited wind-driven sea observations. Nonlinear Processes Geophys., 12, 1011-1020. 